pour tout couple $z_{1}, z_{3} \in E$ équivaut dans cette notation à la condition $f(E) \cdot[f(E)]^{\circ}=0$

L'ensemble $f(E)$ étant ouvert et connexe, il existe en vertu du th. 8 un ensemble $Y \subset S_{2}$ ouvert, simplement connexe, tel que $f(E) \subset Y$ et que $Y \cdot Y^{*}=0$. Soit $f_{1}(z)$ la représentation conforme de $E$ sur $Y$, telle que $f_{1}(0)=0$. Le lemme étant déjà etabli dans le cas des fonctions univalentes, on a $\left|f_{1}^{\prime}(0)\right| \leqslant 1$.

Considérons la fonction $g(z)=f_{1}^{-1}[f(z)]$, qui n'est certainement pas univalente. On a $g(0)=0$ et $|g(z)|<1$ pour tout $z \in E$. En vertu du lemme de Schwartz, on a donc $\left|g^{\prime}(0)\right|<1$. Or, $g^{\prime}(0)=\frac{f^{\prime}(0)}{f_{1}^{\prime}(0)}$, puisque $f(0)=0$. On en tire $\left|f^{\prime}(0)\right|<f_{1}^{\prime}(0)$ et finalement $\left|f^{\prime}(0)\right|<1$, e. q. f. d.

\section{Sur l'intégration des coefficients différentiels d'ordre supérieur.}

Par

\section{Arnaud Denjoy (Paris).}

\section{Introduction.}

1. Soit $f(x)$ une fonction continue définie sur un segment $a b$ $\{a \leqslant x \leqslant b)$. Selon une défnition particulièrement commode, nous dirons que $f(x)$ possède au point $x$ une différentielle d'ordre $n$ si $x+h$ appartenant à $a b, f(x+h)$ est la somme d'un polynome en $h$ et d'un infiniment petit d'ordre supérieur à $n, h$ étant l'infiniment petit principal.

Le coefficient de $\frac{h^{n}}{n !}$ dans le polynome, soit $f_{n}(x)$, sera appelé le $n^{\ominus}$ quotient différentiel de $f$ au point $x$, et le produit $h^{n} f_{n}(x)$ recerra le nom de différentielle $n^{\ominus}$ de $f$ au point $x$.

Le principal problème que nous avons en vue est d'examiner si et dans quelle mesure $f(x)$ est déterminée par son $n^{\mathrm{e}}$ quotient différentiel, puis d'effectuer le calcul de $f(x)$ supposant connu $f_{n}(x)$.

2. D'après la définition adoptée, $f(x)$ admet à fortiori une différentielle de tout ordre entier $p$ si $1 \leqslant p \leqslant n$, et l'égalité

$$
\begin{aligned}
f(x+h)= & f(x)+h f_{1}(x)+\ldots+\frac{h^{p}}{p !} f_{p}(x)+\ldots \\
& \ldots+\frac{h^{n}}{n !}\left[f_{n}(x)+\varepsilon(x, h)\right]
\end{aligned}
$$

détermine, pour $h$ non nul, un nombre $\varepsilon(x, h)$ tendant vers 0 avec $h$, $x$ demeurant invariable.

Fundamenta Mathematicae. T. XXv. 
Dans le cas particulier où $f(x)$ admet des dérivées continues des $(n-1)$ premiers ordres $f^{\prime}, f^{\prime \prime}, f^{(n-1)}$ et une dérivée du $n^{\Theta}$ ordre $f^{(n)}(x), f(x)$ possède une différentielle du $n^{0}$ ordre, et duns l'égalité (1), $f_{p}(x)=f^{(p)}(x)$.

La réciproque n'est pas exacte, dès que $p \geqslant 2 \cdot(7,7 \mathrm{~b})$.

La condition (1) toutefois entraîne la continuité de $f(x)$ et l'existence de sa dérivée première $f_{1}(x)$ au point considéré $x$.

3. On peut $(10-13)$ considérer des différentielles $n^{\text {6s }}$ spécialcs à un ensemble $H$, sur lequel se déplace indifféremment le point $x+h$, simplement assujetti à tendre vers $x$, point d'accumulation de $H$.

Ou bien l'ensemble $H$ est indépendant de $x$. Nous le supposerons généralement parfait, et parcouru par $x$. Ou bien $H$ peut dépendro de $x$, et possède un caractère déterminant pour une différentiolle de $f$ spéciale à un ensemble de la même classe. A cette espèee appartiennent les différentielles approximatives (11b), prépondérantes, unilatérales $(12)$.

Enfin, s'il existe une différentielle d'ordre $n-1$, mais non pas une d'ordre $n$, on peut considérer au lieu d'un quotient différentio d'ordre $n$, limite unique finie d'un certain rapport, des coefficients différentiels extrêmes d'ordre $n$ qui sont les plus grande et plus petite limites finies ou infinies de ce même rapport (13).

4. Grâce à la formule d'interpolation de Lagrange donnant le polynome en $x$ d'ordre $n$ prenant en $n+1$ points $x$ les mômes valeurs que $f(x)$, on établit des relations entre les quotients difflérentiels en deux points distincts $x, x+k(\gamma-9)$.

J'en déduis le théorème essentiel II (14) que, sur tout ensemble parfait $P$ où le nombre $\varepsilon_{n}(x, h)$ est uniformément borné indépendamment de $x$, les $(n-1)$ premiers quotients différentiels sont continus sur $P$ et le $p^{\ominus}$ est différentiable $(n-p-1)$ fois spéciclement dे $P$.

La même conclusion subsiste si l'ensemble-base $H$, relativement auquel la différentiation fondamentale est effectuée, est nou pas le continu, mais un ensemble parfait d'indice fini, observation qui confirme l'intérêt de cette notion d'indice qui s'introduit dans l'intégration des nombres dérivés seconds généralisés de Riemann (15)

$\mathrm{Au}$ contraire, si l'ensemble-base de la différentiation est un ensemble parfait quelconque $H$, seule la continuité sur $P$ (inclus dans $H)$ des quotients différentiels de tout ordre subsiate $(17, b, c)$, mais non pas le fait que le quotient d'ordre $p(1 \leqslant p)$ admette spécialement à $P$ une différentielle d'ordre $n-p$ dont les quotients sont empruntés à ceux de $f$ par rapport à $H$.

Je donne un exemple du fait contraire (19).

Cependant (théorème IV, $\mathrm{n}^{0} 18$ ) le $n^{\theta}$ quotient différentiel de $f(x)$ spécial à un ensemble parfait $H$ est une fonction de classe quel que soit $H$.

Les théorèmes $\mathrm{V}$ et VI précisent comment, les quotients différentiels $f_{p}(x)$ n'étant pas nécessairement ccntinus sur la totalité de $a b$, cependant tout ensemble parfait (continu ou discontinu) contient une purtion où, spécialement à $P$, chacun des $f_{p}$ est continu et différentiable (20-22).

Enfin, l'énoncé du théorème II subsiste encore si l'existence d'un quotient différentiel $n^{\ominus}$ fini en tout point de l'ensemble parfait $P$ fait place à l'hypothèse plus large de l'existence de deux quotients différentiels extrêmes finis, pour un côté au moins (théorème VIII $\mathrm{n}^{0}$ 24).

D'ailleurs, le théorème VII $(23,25-30)$ énonce que la fonction possède une différentielle générale $n^{\ominus}$ sur une pleine épaisseur de l'ensemble $I$, où d'un côté au moins elle a ses coefficients différentiels $n^{\text {es }}$ finis.

5. Etendant la notion de la dérivée seconde généralisée de Riemann, j'étudie (33-48) les quotients différentiels généralisés.

Les $\alpha_{i}$ étant des nombres indépendants de $h$, je considère une combinaison aux différences $Q_{n}\left(f, x, h, \alpha_{1}, \ldots, \alpha_{n+1}\right)$ qui est une somme $n ! \sum \frac{f\left(x+\alpha_{i} h\right)}{\varphi^{\prime}\left(\alpha_{i}\right)}$ infiniment petite avec $h$ et équivalente à $f_{n}(x) h^{n}$ quand $f$ admet un quotient différentiel $n^{e}$ continu $f_{n}(x)$.

Si $R_{n}\left(f, x, h, \alpha_{i}\right)=\frac{Q_{n}}{h^{n}}$ tend vers une limite $g_{n}(x)$ quand $h$ tend indifféremment vers $0, g_{n}(x)=g_{n}\left(f, x, \alpha_{1}, \ldots, \alpha_{n+1}\right)$ est appelé un quotient différentiel $n^{\mathbf{e}}$ généralisé.

$g_{n}(x)$ comme $Q_{n}$ est indépendant de l'addition à $f(x)$ d'un polynome de degré $n-1$ en $x$.

Si $g_{n}(x)$ est donné, peut-on a priori en déduire $f(x)$, sans ambiguité (à l'addition près d'un polynome d'ordre $n-1$ )? Ou, sous une autre forme, équivalente à la précédente, si $g_{n}(x)=0, f(x)$ se réduit-il nécessairement à un polynome unique? 
Il n'en est rien si tous les $\alpha_{t}$ sont de même signe (35) ni ai les $\alpha$ sont symétriques par rapport à zéro, dès que $n>2(44)$.

Toutefois $(36-38)$ quels que soient les $\alpha_{l}$, l'ensemble $E$ (fermé) des points autour desquels $f(x)$ ayant le quotient différentiel $n^{\ominus}$ généralisé 0 ne coïncide pas avec un polynome unique, est non dense (sur le continu).

Je dis que la combinaison $Q_{n}$ est caractéristique si le quotient généralisé correspondant $g_{n}(x)$ ne peut être 0 quand $f$ est égal à deux polynomes (d'ordre $n-1$ au plus) distincts dans deux intervalles séparés par une extrémité commune $(39 \mathrm{~b})$. L'ensemble $E$, s'il existe, est alors parfait, car il ne peut avoir de point isolé. Je n'ai pu démontrer que si la combinaison $Q_{n}$ est caractéristique, $E$ n'existe pas (48). Ce n'est que si l'existence de $E$ est impossible qu'il peut y avoir un procédé opératoire permettant de remonter de $g_{n}(x)$ à $f(x)$.

Si l'un des $\alpha_{i}$ est seul de son signe, la cumbinaison $Q_{n}$ est caractéristique (42).

6. J'étudie enfin (49-65) le calcul d'une fonction $f(x)$ douée en tout point de $a b$ d'un $n^{\ominus}$ quotient différentiel général $f_{n}(x)$, quand on suppose celui-ci connu.

Mais l'exposé gagne en simplicité si l'on élargit les hypothèses. Il suffit de supposer que $f(x)$ est résoluble d'ordre $n$.

La définition de ce caractère (51) implique $1^{\circ}$ des propriétés locales, savoir la continuité de $f(x)$, et d'autro part une condition empêchant que deux polynomes différents d'ordre $n-1$ au plus puissent être ajoutés à $f(x)$ de part et d'autre d'un méme point, sans que $f$ cesse d'être $n$ fois résoluble; $2^{0}$ des propriétés globales relatives à un ensemble parfait quelconque $P$. $P$ doit contenir une portion $P_{1}$ où les propriétés énoncées dans le théorème II sont vérifiées jusqu'à l'ordre $n-1$, le $(n-1)^{\mathrm{e}}$ quotient différentiel spécial à $P_{1}$ étant résoluble (d'ordre 1 ).

Ces hypothèses entraînent l'existence pour $f$ d'un quotient différentiel approximatif $n^{e} f_{n, a}(x)$ sur une épaisseur pleine (52). Réciproquement $f$ est déterminé (à l'addition près d'un polynomo d'ordre $n-1)$ par la connaissance de $\varphi_{n}(x)$ égal à $f_{n, a}(x)$ sur uno épaisseur pleine (56).

Je donne (58-64) le procédé totalisant permettunt de remonter de $\varphi_{n}(x)$ à $f(x)$.
La méthode s'applique dès que l'on sait qu'en tout point, d'un côté au moins, $f(x)$ a ses deux coefficients différentiels $n^{\text {es }}$ extrêmes finis et que $\varphi_{n}(x)$ est égal à l'un d'eux.

\section{Etude générale de la différenciation d'ordre $\boldsymbol{n}$.}

7. Il est aisé de voir par des exemples qu'une fonction $f(x)$ peut avoir une différentielle $n^{\theta}$ en chaque point, sans qu'aucun des quotients différentiels figurant dans la formule (1), pas même $f_{1}(x)$, soit continu. A fortiori, $f_{2}(x)$ n'est-il pas toujours la dérivée de $f_{1}(x)$, ni $f_{p+1}(x)$ celle de $f_{p}(x) \quad(p=1, \ldots, n-1)$.

En effet, soit par exemple $f(x)=x^{n+a} \sin \frac{1}{x^{p}}$ pour $x \neq 0$, ( $n$ entier $\geqslant 2,0<\alpha<1$ ) avec $f(0)=0$.

Pour $x \neq 0, f(x)$ est analytique et par suite admet une différentielle de tout ordre donné, les quotients différentiels étant égaux aux dérivées de même ordre.

Pour $x=0, f(x)$ admet une différentielle d'ordre $n$, et tous les $n$ premiers coefficients différentiels sont nuls.

Or, si $p \geqslant n+\alpha-1, f_{1}(x)=f^{\prime}(x)$ est discontinu à l'origine. En sorte que les dérivées d'ordre au moins égal à 2 n'existent pas à l'origine. Les $n$ premiers quotients différentiels, bien qu'existant en tout point $x$, sont donc discontinus à l'origine.

$7 \mathrm{~b}$. On peut réaliser des circonstances plus générales analogues à celle-ci. Soit $\alpha \beta$ un intervalle de l'axe réel $(\alpha<\beta), \alpha$ et $\beta$ pouvant être infinis $(\alpha=-\infty, \beta=+\infty)$. Par définition, la distance $u$ de $x$ au couple $(\alpha, \beta)$ est le plus petit des deux nombres $|x-\alpha|,|x-\beta|$. Supposons $x$ intérieur à $\alpha \beta$. Nous appellerons pseudo-distance (ou encore distance analytique de $x$ au couple $(\alpha, \beta)$ le nombre $d$ défini par

On a

$$
\frac{1}{d}=\frac{1}{x-\alpha}+\frac{1}{\beta-x} \quad \text { ou } \quad . d=\frac{(x-\alpha)(\beta-x)}{\beta-\alpha} .
$$

$$
\frac{1}{2}<\frac{d}{u}<1, \quad \lim _{u=0} \frac{d}{u}=1, \quad . \quad d=u \quad \text { si } \quad \beta-\alpha=\infty .
$$

Mais $d$ est analytique en $x$ sur l'intervalle $\alpha \beta$.

Soit $E$ un ensemble fermé. Nous définissons comme il suit la pseudo-distance $d(x, E)$ de $x$ à $E:$ Si $x$ est sur $E, d(x, E)=0$. 
Si $x$ est dans un intervalle $\alpha \beta$, fini ou infini, contigu (s'il est fini) ou semi-contigu (s'il est infini) à $E, d(x, E)$ est la pseudo-distance de $x$ au couple $\alpha \beta$. Done, quel que soit $\xi$ sur $E$, on a $|x-\xi| \geqslant d(x, E)$.

Ces notations étant fixées, considérons un ensemble fermé $L$ non dense. Si $x$ est sur $E$, posons $f(x)=0$. Si $x$ est étranger à $E$, soit $f(x)=[d(x, E)]^{n+\alpha} \sin \frac{1}{[d(x, E)]^{p}}(n>2,0<\alpha<1, p \geqslant n+\alpha-1)$.

Quel que soit le point $\xi$ sur $E,|f(x)| \leqslant|x-\xi|^{n+\alpha}$. Dune $f(x)$ admet en tout point une différentielle d'ordre $n$. Les quotients différentiels des $n$ premiers ordres sont tous nuls sur $E$. Ils sont discontinus en chaque point de $E$ ( $E$ étant non-dense). Observons que, spécialement à $E$, chacun d'eux est une fonction continue sur $E$.

Nous retrouveruns dans le cas le plus général des circonstances rappelant celles-ci.

8. Formules auxiliaires. Voici diveršes remarques très élémentaires qui nous seront d'une grande utilité. Soit

$$
u=a_{0}+a_{1} h+\ldots+a_{n} h^{n},
$$

les coefficients $a_{p}$ étant indépendants de $h$.

Donnons à $h$ les valeurs $h_{1}, h_{2}, \ldots, h_{n+1}$ et soient $u_{1}, u_{2}, \ldots, u_{t+1}$ les valeurs correspondantes de $u$. Done

$$
u_{i}=a_{0}+a_{1} h_{i}+\ldots+a_{n} h_{l}^{n}
$$

Posons $\varphi(h)=\left(h-h_{1}\right) \ldots\left(h-h_{n+1}\right)$ et désignons par $s_{h, q}$ la somme des produits $q$ à $q$ des nombres $h_{1}, h_{2}, \ldots, h_{t-1}, h_{l+1}, \ldots, h_{n-1-1}$.
D'après la formule de Lagrange,

$$
\begin{gathered}
a_{0}+a_{1} h+\ldots+a_{n} h^{n}= \\
=\sum_{i=1}^{i=n+1} \frac{u_{i}}{\varphi^{\prime}\left(h_{t}\right)}\left(h-h_{1}\right)\left(h-h_{2}\right) \ldots\left(h-h_{t-1}\right)\left(h-h_{i+1}\right) \ldots\left(h-h_{n+1}\right),
\end{gathered}
$$
d'où

$$
a_{p}=(-1)^{n-p} \sum u_{i} \frac{s_{i, n-p}}{\varphi^{\prime}\left(h_{i}\right)} \quad(p=0,1, \ldots, n) .
$$

$\alpha$ étant un nombre positif donné, considérons des couples de points $\left(y, y^{\prime}\right)$ liés par les relations

$$
\begin{gathered}
f\left(y^{\prime}\right)=f(y)+\left(y^{\prime}-y\right) A_{1}(y)+\ldots \\
\ldots+\frac{\left(y^{\prime}-y\right)^{n-1}}{(n-1) !} A_{n-1}(y)+\frac{\left(y^{\prime}-y\right)^{n}}{n !}\left[A_{n}(y)+\delta \alpha\right] \quad \delta^{2}<1,
\end{gathered}
$$

$A_{1}(y), \ldots, A_{n-1}(y), A_{n}(y)$ étant indépendants de $y^{\prime} ; \delta$ dépend de $y, y^{\prime}, n, \alpha$.

9. Thêrème $\boldsymbol{I}$. Si les relations (4) sont vérifiées quand on y remplace indifféremment $y$ par $x$ ou par $x+k$, et $y^{\prime}$ par $x+k+h_{i}$ $(i=1,2, \ldots, n+1)$, si d'autre part, $\frac{1}{\left|h_{i}-h_{j}\right|}<\frac{B}{\left|k_{i}\right|}(i \neq j),\left|h_{i}\right|<C|k|$, $B$ et $C$ étant indépendants de $i, j, k$, on a les relations.

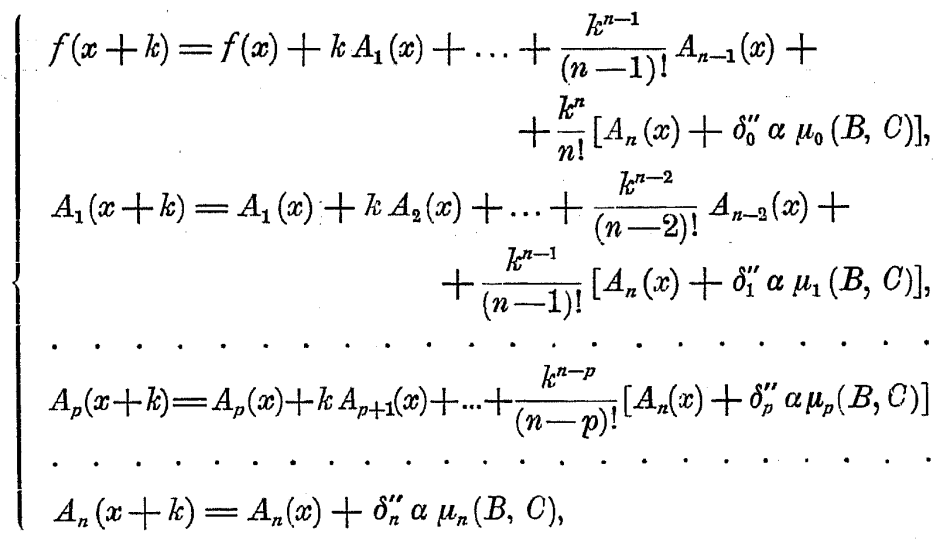

les $\mu_{p}(B, C)(p=0,1, \ldots, n)$ dépendant seulement de $B, C, n, p$, mais non pas de $x, k, \alpha$, et les $\left|\delta_{p}^{\prime \prime}\right|$ étant inférieurs $\grave{a} 1$.

\section{On a par hypothèșe}

(6)

$$
\left\{\begin{array}{c}
f\left(x+k+h_{i}\right)=f(x)+\left(k+h_{i}\right) A_{1}(x)+\frac{\left(k+h_{i}\right)^{2}}{2} A_{2}(x)+\ldots \\
\ldots+\frac{\left(k+h_{i}\right)^{n-1}}{(n-1) !} A_{n-1}(x)+\frac{\left(k+h_{i}\right)^{n}}{n !}\left[A_{n}(x)+\delta_{i} \alpha\right] \\
f\left(x+k+h_{i}\right)=f(x+k)+h_{i} A_{1}(x+k)+\frac{h_{i}^{2}}{2} A_{2}(x+k)+\ldots \\
\ldots+\frac{h_{i}^{n-1}}{(n-1) !} A_{n-1}(x+k)+\frac{h_{i}^{n}}{n !}\left[A_{n}(x+k)+\delta_{i}^{\prime} \alpha\right]
\end{array}\right.
$$

avec $\delta_{i}^{2}, \delta_{l}^{2}<1$. 
Done, si nous posons

$$
\begin{array}{r}
A_{p}(x+k)-A_{p}(x)-k A_{p+1}(x)-\ldots-\frac{k^{n-p}}{(n-p) !} A_{n}(x)=p ! a_{p} \\
(p=0,1.2, \ldots, n)
\end{array}
$$

la relation

$$
0=a_{0}+h a_{1}+h a_{2}+\ldots+h^{n} a_{n}+\left[\delta^{\prime} \frac{h^{n}}{n !}-\delta \frac{(k+h)^{n}}{n !}\right] \alpha
$$

est vérifiée avec $|\delta|,\left|\delta^{\prime}\right|<1$ pour $h=h_{1}, h_{2}, \ldots, h_{n+1}$.

En posant $u_{i}=\left[-\delta^{\prime} \frac{h_{i}^{n}}{n !}+\delta_{i} \frac{\left(k+h_{t}\right)^{n}}{n !}\right] \alpha$, nous trouvons les égalités (2). Appliquons la formule (3). D'après

$$
\left|u_{i}\right|<\frac{\alpha}{n !}\left|k_{i}\right|^{n}\left[C^{n}+(C+1)^{n}\right], \quad\left|\frac{s_{t, n-p}}{\varphi^{\prime}\left(h_{i}\right)}\right|<\frac{(n+1) !}{p !(n-p) !} \frac{B^{n} C^{n-p}}{\left|k_{k}\right|^{p}}
$$

un calcul immédiat donne

$$
p !\left|a_{p}\right|<\frac{n+1}{(n-p) !}\left|k^{n-p}\right| 2 \alpha B^{n}(C+1)^{n} C^{n-p} .
$$

Le théorème est donc établi avec

$$
\mu_{p}(B, C)=2(n+1) B^{n}(C+1)^{n} C^{n-p}
$$

Cette valeur de $\mu_{p}$ pourrait être améliorée, mais une précision supplémentaire n'offrirait aucun intérêt pour la suite. Avant de tirer de ce théorème les conséquences qu'il comporte, nous allons étendre la notion de différentielle $n^{\mathrm{e}}$.

10. Différentielles spéciales. Soit $H$ un ensemble linéaire, situé sur l'axe des nombres réels $x$, et $f(x)$ une fonction définie sur $H$. Supposons $x$ commun à $H$ et à son dérivé.

Nous dirons qu'au point $x, f(x)$ admet spécialement d $H$ une différentielle d'ordre $n$, si $x+h$ décrivant $H, f(x+h)$ est la somme d'un polynome en $h$ et d'un infiniment petit d'ordre supérieur à $n$ par rapport à $h$ pris pour infiniment petit principal. Il existe donc $n$ nombres $f_{p}(x, H)(p=1, \ldots, n)$ tels que $\varepsilon(x, h)$ défini par

$$
\text { (8) } f(x+h)=f(x)+h f_{1}(x, H)+\frac{h^{2}}{2} f_{2}(x, H)+\ldots+\frac{h^{n}}{n !}\left[f_{n}(x, H)+\varepsilon(x, h)\right]
$$

tend vers 0 avec $h$ si le point $x+h$ ne cesse de se déplacer sur $H$. $f_{n}(x, H)$ est appelé le $n^{\ominus}$ quotient différentiel de $f$ spécial à $H$ au point $x$.

$h^{n} f_{n}(x, H)$ est la différentielle $n^{\ominus}$ de $f$ spéciale à $H$ au point $x$.

Ici encore, l'existence de la différentielle $n^{\mathrm{a}}$ spéciale à $H$ entraîne l'existence de toutes les différentielles d'ordre inférieur à $n$, spéciales à $H$.

Si $f(x)$ admet au point $x$ une différentielle $n^{\mathbf{\theta}}$ spécialement à $H$, celle-ci est déterminée par la connaissance de $f$ sur une suite particulière quelconque $x+h_{1}, x+h_{2}, \ldots, x+h_{m}, \ldots$ de points de $H$ tendant vers $x$, quand $m$ croît.

En effet, l'égalité (8) entraîne successivement

$$
\begin{gathered}
f(x)=\lim _{m=\infty} f\left(x+h_{m}\right) \\
f_{1}(x, H)=\lim _{m=\infty} \frac{f\left(x+h_{m}\right)-f(x)}{h_{m}} \\
f_{2}(x, H)=\lim _{m=\infty} 2 \frac{f\left(x+h_{m}\right)-f(x)-h_{m} f_{1}(x, H)}{h_{m}^{2}}
\end{gathered}
$$

Si $f(x)$ possède une différentielle spécialement à un ensemble $H_{1}$ contenant $H$, a fortiori possède-t-elle, spécialement à $H$, une différentielle du même ordre, et avec les mêmes quotients différentiels jusqu'à cet ordre inclus.

En particulier, si $f(x)$ possède au point $x$ une différentielle d'ordre $n$ relative au continu, ulors, quel que soit $H, f_{n}(x, H)$ existe et est identique à $f_{n}(x)$. Nous appellerons encore ordinaire ou générale (c'est-à-dire commune à tous les ensembles admettant $x$ pour point d'accumulation) la différentielle $n^{\ominus}$ relative au continu.

11. A priori, il y aura deux façons d'envisager une différentielle $n^{\ominus}$ spéciale de $f$ au point $x$.

Ou bien on considérera un ensemble $H$ indépendant de $x$, et sur lequel $x$ se déplace, et en chaque point duquel $f$, spécialement à $H$, admet une différentielle $n^{\ominus}$. Ce cas donne l'extension la plus simple de la différentielle $n^{\mathrm{e}}$ ordinaire.

Ou bien l'ensemble $H$, spécialement auquel $f$ est $n$ fois différentiable au point $x$, existe pour chaque position de $x$ (tout au moins pour tout $x$ appartenant à un certain ensemble linéaire $\omega$ ), mais $H$ varie avec $x$. $B$ est un ensemble $H(x)$. 
$x$ décrivant $\omega$, il pourra se faire qu'il n'existe pas d'ensemble $H(\omega)$ commun à tous les $H(x)$.

Mais il sera indispensable, pour mettre en évidence des propriétés de la $n^{\theta}$ différentielle de $f$ au point $x$ spécialoment à $H(x)$, de supposer que ce dernier ensemble vérifie des conditions limitant sa nature, indépendamment de $x$ variable sur $\omega$.

Par exemple, $H(x)$ a au point $x$, l'épaisseur 1 , ou une épaisseur bilatérale supérieure à $1 / 2$, ou $H(x)$ est, dans un intervalle assez court entourant $x$, un résiduel du continu (complémentaire d'un ensemble de première catégorie), etc. On peut espérer que, $x^{\prime}$ étant sur $\omega$ assez près de $x$, et sous certaines conditions assez générales, deux ensembles $H(x), H\left(x^{\prime}\right)$ auront des points communs formant un ensemble de mesure positive, un résiduel d'intervalle, etc...

$f$ peut posséder au point $x$ diverses différentielles d'urdre $n$, correspondant à des ensembles $H^{\prime}, H^{\prime \prime}, \ldots$ différents admettant chacun $x$ pour point limite. Bien entendu, il faut pour cela que l'ensemble $H^{\prime} \cdot H^{\prime \prime}$ commun à deux ensembles quelconques $H^{\prime}, H^{\prime \prime}$ spécialement auxquels $f$ a des différentielles $n^{\text {es }}$ inégales n'admette pas $x$ pour point limite.

Cette dernière circonstance sera impossible si par exemple l'épaisseur inférieure de $H^{\prime}$ et de $H^{\prime \prime}$ d'un même côté de $x$ surpasse 1/2 (ou encore si $H^{\prime}$ et $H^{\prime \prime}$ sont les complémentaires d'ensembles de première catégorie au voisinage de $x$ ).

$11 \mathrm{~b}$. Une différentielle d'ordre $n$ de $f(x)$ sera dite approximative (bilatérale, droite, gauche) si elle est spéciale à un ensemble $H(x)$ d'épaisseur 1 (bilatérale, droite, gauche) au point $x$. Il existe alors des nombres $f_{1, \alpha}(x), f_{2, \alpha}(x), \ldots, f_{n, \alpha}(x)$ tels que $\varepsilon(x, h)$ défini par

$\left(8^{\mathrm{bis}}\right) f(x, h)=f(x)+h f_{1, \alpha}(x)+\frac{h^{2}}{2} f_{2, \alpha}(x)+\ldots+\frac{h^{n}}{n !}\left[f_{n, \alpha}(x)+\varepsilon(x, h)\right]$

tend vers 0 avec $h$ quand le point $x+h$ décrit un certain ensemble $H(x)$ d'épaisseur (qualifiée ou bilatérule) égale à 1 au point $x$.

12. Quand tous les points de $H(x)$ distincts de $x$ sont d'un même côté de $x, h$ a un signe constant, les différentielles, les quotients différentiels sont dits unilatéraux: droits pour $h>0$, gauches pour $h<0$.

Nous dirons que $f$ admet au point $x$ une différentielle prépondérante (bilatérale, ou droite, ou gauche) définissant des quotients différentiels prépondérants au même point (et de même sorte) si $f$ admet une différentielle spéciale à un ensemble d'épaisseur (bilatérale, droite ou gauche) supérieure à $1 / 2$ au point $x$.

$\mathrm{Si}$ la différentielle prépondérante de $f$ existe au point $x$, cette différentielle est déterminée, d'après une remarque faite un peu plus haut. Au contraire, a priori une fonction $f(x)$ pourrait avoir pour un côté donné $p$, mais non pas $(p+1)$ différentielles $n^{\text {es }}$ distinctes, chacune étant spéciale à un ensemble d'épaisseur inférieure $e_{t}$ (du côté considéré) si $\frac{1}{p+1}<e_{i} \leqslant \frac{1}{p}$ au point $x$.

13. Enfin, supposons simplement que $f(x)$ admette une différentielle d'ordre $n-1$ au point $x$, spéciale à un ensemble $H$ admettant $x$ pour point limite. Soit $\Phi_{n}(x, h)$ défini par

(9) $f(x+h)=f(x)+h f_{1}(x, H)+\ldots+\frac{h^{n-1}}{(n-1) !} f_{n-1}(x, H)+\frac{h^{n}}{n !} \Phi_{n}(x, h)$.

Nous donnerons le nom de coefficients différentiels $n^{\mathrm{es}}$ supérieurs, inférieurs (l'un et l'autre étant dits eitrềnes), moyens de $f$ au point $x$ spécialement à $H$, à la plus grande limite, à la plus petite limite, à l'une quelconque des valeurs limites de $\Phi_{n}(x, h), h$ tendant vers 0 , de façon que $x+h$ se déplace indifféremment sur $B$.

Si $H$ est le continu, les quotients différentiels $n^{\text {es }}$ extrêmes et moyens de $f$ au point $x$ ne sont pas qualifiés, ou sont dits ordinaires, généraux.

Comme application de ces définitions et du théorème $I$, nous établirons la proposition suivante:

14. Théorème $\boldsymbol{I T}$. $f(x)$ admettant une différentielle $n^{\ominus}$ ordinaire en tout point $x$ du segment $a b$, et $\varepsilon(x, h)$ étant le nombre défini par l'égalité (1), si $P$ est un ensemble parfait situé sur $a b$, auquel sont associés deux nombres positifs $\alpha, \eta$ indépendants de et tels que, pour tout point $x$ de $P$, l'inégalité $|h|<\eta$ entraîne $|\varepsilon(x, h)|<\alpha$,

sous ces conditions suffisantes.

$1^{0} f_{n}(x)$ est borné sur $P$ et, $\mu$ étant un nombre indépendant de $x$ et de $\alpha$, l'oscillation de $f_{n}(x)$ en chaque point de $P$ et spécialement $\grave{a} P$ est inférieure à $\mu \alpha$;

$2^{0} f_{n-1}(x)$ est continu sur $P$, spécialement $\grave{a} P$, et, en chaque point de $P$, les nombres dérivés extrêmes de $f_{n-1}(x)$ spéciaux à $P$ sont bornés et écartés de moins de $2 \mu \alpha$; 
$3^{0}$. spécialement à $P$, en chaque point de $P$, et pour $p=1,2, \ldots, n-2$,

a) $f_{p}(x)$ est continu, admet une différentielle d'ordre $n-p-1$ spéciale à $P$,

b) le $m^{\Theta}$ quotient différentiel $\left[f_{p}\right]_{n t}(x, P)$ de $f_{p}(x)$ spécial d̀ $P$ est égal d̀ $f_{p+m}(x)$ pour $m=1,2, \ldots, n-p-1$.

Les formules (5) fournissent immédiatement ce théorème, quand on remplace dans (5) $A_{p}(x), A_{p}(x+k)$ par $f_{p}(x), f_{p}(x+k)$.

En effet, soient $x$ et $x+k$ deux points de $P$ avec $|k|<\eta$. Donnonsnous une suite de nombres positifs décroissants $\beta_{1}<1, \beta_{2}, \ldots, \beta_{n+1}$, indépendants de $x$ et de $k$, (on pourrait prendre $\beta_{l}=1-\frac{i}{n+2}$ ). Posons $h_{i}=-k\left(1-\beta_{i}\right)$ et $k_{i}=k+h_{i}=k \beta_{i}$. Le point $x+k+h_{i}=$ $=x+k_{i}=x+k \beta_{i}$ est compris entre $x$ et $x+k$. Donc $\left|h_{l}\right|,\left|k_{l}\right|$ et $\left|k+h_{l}\right|$ sont inférieurs à $\eta$. La formule (5) s'applique on y remplaçant $A_{p}(x), A_{p}(x+k)$ par $f_{p}(x), f_{p}(x+k), B$ par le plus grand des nombres $\frac{1}{\beta_{i}-\beta_{i+1}}, C$ par 1 (et aussi $C+1$, dans le dernier facteur par 1). Dès lore, $\mu_{p}(B, C)=2(n+1) B^{n}=\mu$ indépendamment de $p$ On a donc

(10) $f_{p}(x+k)=f_{p}(x)+k f_{p+1}(x)+\ldots+\frac{k^{n-p}}{(n-p) !} f_{n}(x)+\delta_{p} \frac{k^{n-p}}{(n-p) !} \mu \alpha, \delta_{p}^{2}<1$ pour $p=0,1, \ldots, n,|k|<\eta$.

Le premier alinéa du théorème résulte de la dernière des formules précédentes

$$
\left|f_{n}(x+k)-f_{n}(x)\right|<\mu \alpha \text { pour }|k|<\eta .
$$

$f_{n}$ est borné sur $P$, puisque, le diamètre de $P$ étant au plus $b-a$, $P$ est divisible en moins de $\frac{b-a}{\eta}$ portions, sur chacune desquelles l'oscillation de $f$ spéciale à $P$ est inférieure à $\mu \alpha$.

Toutes les autres parties de l'énoncé découlent dès lors des formules (10) pour $p=n-1$ d'abord, et pour $p=0,1, \ldots, n-2$ ensuite.

15. Le théorème II (et par suite ausssi III) subsiste quand il s'agit d'une différentielle $n^{\mathrm{e}}$ de $f(x)$ spéciale à un ensemble parfait donné $H$, si, outre l'hypothèse que, $P$ étant parfait situé sur $H$, la double condition $|h|<\eta$ et $x+h$ sur $H$, entraîne dans la formule (1): $|\varepsilon(x, h)|<\alpha$, quel que suit $x$ sur $P$, si par sureroît on suppose que l'indice de $H$ est uniformément borné aux points de $P$.

J'ai intruduit la notion d'indice d'un ensemble parfait $H$ à l'occasion de mes études sur l'intégration des dérivées secondes généralisées. L'indice de $H$ èn un de ses points $\xi$ est la borne inférieure $\omega \geqslant 1$ des nombres $\lambda$ tels qu'il existe une suite

$$
\xi+k_{0}, \xi+k_{1}, \ldots, \xi+k_{n}, \ldots
$$

située sur $H$ et tendant vers $\xi$, avec $1<\left|\frac{k_{n}}{k_{n+1}}\right|<\lambda$.

Si aucun nombre $\lambda$ n'existe vérifiant cette condition, l'indice de $H$ au point $\xi$ est infini.

Nous disons que l'indice de $H$ est uniformément borné par $\gamma \geqslant 1$ sur $P . \gamma$ étant indépendant de $\xi$, si dans la condition précédente on peut faire $\lambda=\gamma$ et $\left|k_{0}\right|>\theta, \quad \theta$ étant indépendant de $\xi$.

On peut alors écrire les formules (6), $x$ et $x+k$ étant deux points de $P$, si $|k|<\frac{\eta}{2},\left|h_{i}\right|<\frac{\eta}{2}$, en remplaçant dans (6)

$A_{1}(x), \ldots, A_{p}(x), \ldots, A_{p}(x+k), \ldots \quad$ par $\quad f_{1}(x, H), \ldots, f_{p}(x, H) f_{p}(x+k, H), \ldots$,

$$
(p=1, \ldots, n) \text {. }
$$

Nous observons que l'on peut trouver une suite de nombres positifs ou négatifs décroissants en valeur absolue, $\beta_{1}, \beta_{2}, \ldots, \beta_{n+1}$, avec $\left|\beta_{1}\right|<1$, mais dépendant de $x$ et de $x+k$, de façon que

$1^{0}$ si $h_{i}=-k\left(1-\beta_{i}\right)$, le point $x+k_{i}=x+h+k_{i}=x+\beta_{i} k$ appartienue à $B$,

$2^{0} \frac{1}{\left|\beta_{i}-\beta_{i+1}\right|}$ et $\frac{1}{\left|\beta_{i}-\beta_{i+2}\right|}$ soient bornés par un nombre $B$ indépendant de $x$ et de $k$.

En effet, on peut trouver $\beta_{1}$ tel que $x+\beta_{1} k$ soit sur $H$ avec $\frac{1}{\gamma^{2}}<\left|\beta_{1}\right|<\frac{1}{\gamma}, x+\beta_{i} k$ soit sur $H$ avec $\frac{\left|\beta_{i-1}\right|}{\gamma^{2}}<\left|\beta_{i}\right|<\frac{\left|\beta_{i-1}\right|}{\gamma}$, pour $i=1,2, \ldots, n+1$.

Faisons $C=2($ et non $C+1=2), B=\frac{\gamma^{2 n+2}}{\gamma-1}, \mu_{p}=2^{2 n-p}(n+1) \frac{\gamma^{2 n(n+1)}}{(\gamma-1)^{n}}$ $\mu_{p}=\mu_{H} 2^{-p}$. 
Dès lors, a fortiori pour $p=1,2, \ldots, n$

$$
\begin{gathered}
f_{p}(x+k, H)=f_{p}(x, H)+k f_{p+1}(x, H)+\ldots \\
\cdots+\frac{k^{n-p}}{(n-p) !}\left[f_{n}(x, H)+\delta_{p} \mu_{H} \alpha\right.
\end{gathered}
$$

avec $\delta_{p}<1$ si $|k|<\frac{\eta}{2}$.

L'énoncé du théorème II subsiste done, mais la valeur du coefficient $\mu$, multiplicateur de $\alpha$, est autre que si $H$ est le continu.

16. Si au contraire l'indice de $H$ est infini en chacun de ses points, il n'est plus exact que sur un ensemble parfuit $P$ situe sur $H$ et où les hypothèses: $x$ sur $P, x+h$ sur $H,|h|<\eta$ entraî. nent uniformément $|\varepsilon(x, h)|<\alpha$, les quotients différentiels $f_{p}(x, H)$ aient en tout point de $P$ et spécialement à $P$, des différentiolles d'ordre $n-p-1$, ni a fortiori que le $k^{\circ}$ coefficient différentiel spécial à $P$ de $f_{p}(x, H)$, donc $\left[f_{p}(x, H)\right]_{k}(x, P)$, soit égal a $f_{p+k}(x, H)$, même pour $k=1$ (18).

Toutefois, nous allons, même dans le cas le plus général, constater, avec les hypothèses faites, la continuité des coefficients $f_{1}, \ldots, f_{p}(x, H), \ldots$ sur $P$.

17. $\alpha$ et $\eta$ étant deux nombres positifs donnés, nous désignerons par $e_{n}(H, f, \alpha, \eta)$ l'ensemble des nombres $y$ de $H$ en lesquels

$1^{\circ} f$ admet spécialement à $H$ une différentielle d'ordre $n$,

$2^{0}$ les conditions (4) où $A_{p}(y)$ est remplacé par $f_{p}(y, H)$ $(p=1,2, \ldots, n)$ sont vérifiées si $y^{\prime}$ est sur $H$ et si en mémo temps $\left|y^{\prime}-y\right|<\eta$.

Eu somme

(12) $f\left(y^{\prime}\right)=f(y)+\left(y^{\prime}-y\right) f_{1}(y, H)+\ldots+\frac{\left(y^{\prime}-y\right)^{n}}{n !}\left[f_{p}(y, H)+\delta \alpha\right]$

avec $\delta^{2}<1$, moyennant: $y$ sur $e_{n}, y^{\prime}$ sur $H,\left|y^{\prime}-y\right|<\eta$.

Nous dirons encore que, spécialement à $H, f$ est, à $\alpha$ et $\eta$ près, uniformément différentiable à l'ordre $n$ sur $e_{n}(H, f, \alpha, \eta)$.

Nous ne supposons pas a priori $f$ différentiable ni meme continu sur $H$ aux points étrungers à $e_{n}$. Sur $e_{n}$ lui-même l'inégalité (12) n'implique pas par elle-même l'existence d'un quotient différentiel $n$, mais seulement de quotients différentiels extrêmes finis et écartés de moins de $\alpha$.
Théorème III. En tout point $\xi$ de $\bar{e}_{n}(H, f, \alpha, \eta)$,

$1^{0}$ spécialement à $H, f(x)$ est continu t $(n-1)$ fois différentialle,

$2^{0}$ spécialement à $\vec{e}_{n}(H, f, \alpha, \eta)$, les $(n-1)$ premiers quotients différentiels $f_{p}(x, H)$ sont continus, les $n^{\ominus}$ coefficients différentiels extrêmes, $f_{n, i}(\xi, H), f_{n, 1}(\xi, H)$ étant bornés indépendamment de $\xi$, et, en chaque point $\xi$, écartés l'un de l'autre de moins de $2 \alpha$.

Selon l'usage, $\bar{e}_{n}(H, f, \alpha, \eta)$ désigne $e_{n}$ accru de ses points d'accumulation étrangers à lui, ce que l'on appelle encore la fermeture de $e_{n}$.

Le premier alinéa ne demande à être démontré qu'aux points $\xi$ limites de $e_{n}$ et étrangers à lui, donc aux points $\xi$ de $\bar{e}_{n}-e_{n}$, lesquels appartiennent à $e_{n}^{\prime}$.

Soit done $\xi$ un point de $\bar{e}_{n}(H, f, \alpha, \eta)$. Si $\xi$ est étranger à $e_{n}$ nous avons dit que $\xi$ est un point d'accumulation de $e_{n}$ étranger à ce ensemble. A priori nous ne supposons même pas $f$ continu spécialement à $H$ au point $\xi$.

Soient $\xi+h_{1}, \xi+h_{2}, \ldots, \xi+h_{n+1} n+1$ points de $H$, deux à deux distincts, avec $\left|h_{i}\right|<\eta$. Ce choix est possible, $H$ étant parfait et contenant $\xi$, puisque $\xi$ étant dans $\bar{e}_{n}$ est dans $H$.

Soit $\xi+k$ un point de $e_{n}$, assez vuisin de $\xi$ pour que $\left|h_{i}-k\right|<\eta$ pour $i=1,2, \ldots, n+1$. Dans (12) remplaçons $y^{\prime}$ par $\xi+h_{i}$, $y$ par $\xi+k$. Alors

$$
\begin{gathered}
f\left(\xi+h_{i}\right)=f(\xi+k)+\left(h_{i}-k\right) f_{1}(\xi+k, H)+\ldots \\
\ldots+\frac{\left(h_{i}-k_{j}^{n}\right.}{n !}\left[f_{n}(\xi+k, H)+\delta_{i} \alpha\right]
\end{gathered}
$$

avec $\delta_{i}^{2}<1$ pour $i=1,2, \ldots, n+1$. $\delta_{i}$ est une fonction de $\xi$, de $k$ de $h_{i}$, de $\alpha$. La formule (3) nous donne donc:

$$
\frac{1}{p !} f_{p}(\xi+k, H)=\sum_{i=1}^{i=n+1}\left[f\left(\xi+h_{i}\right)-\delta_{i} \alpha \frac{\left(h_{i}-k\right)^{n}}{n !}\right] \frac{S_{t n-p}\left(h_{m}-k\right)}{\varphi^{\prime}\left(h_{t}\right)},
$$

$S_{i \cdot n-p}\left(h_{m}-k\right)$ étant la somme des produits $n-p$ à $n-p$ des nombres $h_{1}-k, h_{2}-k, \ldots . h_{n+1}-k$, sauf $h_{i}-k$.

Lorsque $k$ tend vers $0, \xi+k$ ne quittant pas $e_{n}$, les $h_{i}$ restant invariables, il est évident que $f_{p}(\xi+k, H)$ reste borné (pour $p=0,1, \ldots, n)$. 
$17 \mathrm{~b}$. Soit $\Phi_{p}(\xi)$ une quelconque des limites (plus grande, ou plus petite, ou moyenne) de $f_{p}(\xi+k, H)$ quand $\xi+k$ tend vers $\xi$ (sans quitter $e_{n}$ ). A cette valeur limite correspond un système de nombres $\delta_{i}^{\prime \prime}$ de façon que

$$
\frac{\Phi_{p}(\xi)}{p !}=\sum_{i=1}^{i=n+1}\left[f\left(\xi+h_{i}\right)-\delta_{i}^{\prime \prime} \alpha \frac{h_{i}^{n}}{n !}\right] \frac{S_{i \cdot n-p}\left(h_{m}\right)}{\varphi^{\prime}\left(h_{i}\right)}, \quad \delta_{i}^{\prime \prime 2}<1 .
$$

Soit $\xi+k, \xi+k^{\prime}, \ldots, \xi+k^{(r)}, \ldots$, une suite de points de $e_{n}(H, f, \alpha, \eta)$ tendant vers $\xi$ et tels que pour $p=0,1,2, \ldots, n$, chacun des quotients $f_{p}\left(\xi+k^{(r)}, H\right)$ tende vers une limite unique $\Phi_{p}(\xi)$, nécessairement finie. On a les relations:

(13) $\quad f(\xi+h)=f\left(\xi+k^{(r)}\right)+\left(h-k^{(r)}\right) f_{1}\left(\xi+k^{(r)}, H\right)+\ldots$

$$
\ldots+\frac{\left(h-k^{(r)}\right)^{n}}{n !}\left[f_{n}\left(\xi+k^{(r)}, H\right)+\delta^{(r)} \alpha\right]
$$

$\delta^{(r)}$ dépendant de $\xi$, de $h$, de $k^{(r)}$, mais vérifiant $\left|\delta^{(r)}\right|<1$ sous les conditions suffisantes: $|h|<\eta,\left|h-k^{(r)}\right|<\eta, \xi+h$ sur $H$.

Donc, les deux facteurs de chaque terme du second membre de (13) ayant une limite unique, il en est de même de $\delta^{(r)}$, qui tend vers un nombre $\delta^{\prime \prime}=\delta^{\prime \prime}(\xi, h)$, et

(14) $f(\xi+h)=\Phi(\xi)+h \Phi_{1}(\xi)+\ldots+\frac{h^{n}}{n !} \Phi_{n}(\xi)+\frac{h^{n}}{n !} \delta^{\prime \prime} \alpha, \quad\left|\delta^{\prime \prime}\right| \leqslant 1$, sous les seules conditions qui demeurent: $|h|<\eta, \xi+h$ sur $H$.

17 c. Observons que l'égalité (13) est vraie même pour $h=0$. Il en est donc de même de l'égalité (14), et par suite $\Phi(\xi)=f(\xi)$. D'autre part, l'égalité $(14)$ où $\Phi(\xi)$ est remplacé par $f(\xi)$ signifie qu'au point $\xi$,

$1^{0} f(x)$ admet spécialement à $H$ une différentielle $(n-1)^{\mathrm{e}}$, les $(n-1)$ premiers quotients différentiels $f_{p}(\xi, H)$ élant égaux à $\Phi_{1}(\xi), \ldots, \Phi_{n-1}(\xi)$, et $f(x)$ admet spécialement à $H$ des coefficients différentiels $n^{\text {es }}$ finis compris entre $\Phi_{n}(\xi)-\alpha$ et $\Phi_{n}(\xi)+\alpha$ inclusivement.

$2^{\circ} \mathrm{La}$ relation (14) ne peut pas être vérifiée par une autre suite de coefficients $\Psi_{1}(\xi), \ldots, \Psi_{n-1}(\xi)$ respectivement substitú́s à $\Phi_{1}(\xi), \ldots, \Phi_{n-1}(\xi)$, ni par un coefficient $\Phi_{n}(\xi)$ substitué à $\Phi_{n}(\xi)$ si $\left|\Psi_{n}(\xi)-\Phi_{n}(\xi)\right|>2 \alpha$.
Done a) les nombres $f_{1}(\xi+k, H), f_{2}(\xi+k, H), \ldots, f_{n-1}(\xi+k, H)$ quand $\xi+k$ tend indifféremment vers $\xi$ sans quitter $H$, tendent nécessairemment vers $\Phi_{1}(\xi)=f_{1}(\xi, H), \ldots, \quad \Phi_{n-1}(\xi)=f_{n-1}(\xi, H)$ respectivement. Sinon on pourrait obtenir une seconde relation (14) vérifiée elle aussi avec $\delta^{2}<1$ pour $|h|<\eta, \xi+h$ sur $H$, ce qui est impossible.

Done $f_{p}(x, H)$ est continu sur $\bar{e}_{n}$ pour $p=1,2, \ldots, n-1$.

b) la formule (14) exige que $f_{n}(\xi+k, H), \xi+k$ étant sur $e_{n}$ et tendant vers $\xi$, ait sa plus grande et sa plus petite limite $\Lambda(\xi, H)$ et $\lambda(\xi, H)$ finies, différant entre elles de moins de $2 \alpha$, et que

$$
\Lambda-\alpha \leqslant f_{n, l}(\xi, H) \leqslant f_{n, s}(\xi, H)<\lambda+\alpha .
$$

Il en résulte immédiatement que $f_{n}(x, H)$ est borné sur $e_{n}$ et que les $n^{\text {es }}$ coefficients différentiels extrêmes de $f$ spécialement à $H$ sont bornés sur $\bar{e}_{n}$ et, en chaque point de $\bar{e}_{n}$, diffèrent entre eux de moins de $2 \alpha$.

18. Théorème $\boldsymbol{I V}$. Une fonction $f(x)$ étant définie et continue sur un ensemble parfait donné $H$, le $n^{\mathrm{e}}$ quotient différentiel de $f(x)$ spécial à $H$ est une fonction de classe 1 sur $H$.

Si $H$ est le continu, la proposition résulte de l'égalité immédiatement déduite de (1):

$$
\text { (15) } \frac{1}{n !} f_{n}(x)=\sum_{i=1}^{i=n+1}\left[f\left(x+\alpha_{i} h\right)-\frac{\alpha_{i}^{n}}{n !} h^{n} \varepsilon\left(x, \alpha_{i} h\right)\right] \frac{1}{h^{n} \varphi^{\prime}\left(\alpha_{i}\right)} \text {, }
$$

les $\alpha_{i}$ étant $(n+1)$ nombres distincts indépendants de $x$ et de $h$. On pose $\varphi(u)=\left(u-\alpha_{1}\right) \ldots\left(u-\alpha_{n-1}\right)$.

$$
\text { Donc }
$$

$$
f_{n}(x)=\lim _{h=0} \frac{n !}{h^{n}} \sum_{i=1}^{i=n+1} \frac{f\left(x+\alpha_{i} h\right)}{\varphi^{\prime}\left(\alpha_{i}\right)}
$$

formule qui démontre le théorème.

$18 \mathrm{~b}$. Supposons $H$ parfait quelconque.

En vertu du théorème de Bairé, pour prouver que $f_{n}(x, H)$ est de classe 1 sur $H$, c'est-à-dire est limite de fonctions définies et continues sur $H$, il suffit de prouver l'impossibilité que, sur un ensemble parfait $P$ inclus dans $H, f_{n}(x, H)$ soit totalement discontinue,

Fundamenta Mathematicae. T. XXV. 
ceci entraînant l'existence d'un nombre positif $2 \alpha$ indépendant de $x$, tel qu'en tout point de $P$ l'oscillation de $f_{n k}(x, H)$ spécialement à $P$ surpasse $2 \alpha$.

Nous déduirons très simplement ceci du théorème III. Nous reprenons les notations utilisées dans ce théorème. $e_{n}(H, f, \alpha, \eta)$ est l'ensemble des points $x$ de $H$ tels que les conditions $|h|<\eta$, $x+h$ sur $B$ entrânent dans la formule $(1)|\varepsilon(x, h)|<\alpha$.

$\alpha$ étant donné, tout point $x$ de $H$ appartient à $e_{n}(H, f, a, \eta)$ dès que $\eta$ est assez petit. Cette condition, satisfaite pour toute valeur positive de $\alpha$, est en effet identique à l'hypothèse que $f(x)$ admet en tout point $x$ de $H$ une différentielle $n^{\text {a }}$ spéciale à $H$.

Si done $e_{n^{\prime} p}(\alpha)$ désigne l'ensemble $e\left(H, f, \alpha, \frac{1}{p}\right)$, l'ensemble $e_{n \cdot p}(\alpha)$ croissant avec l'entier $p$ finit par contenir tout point $x$ de $H$ donné d'avance, à partir d'une certaine valeur $p_{0}=p_{0}(x)=p_{0}(x, H)$.

Soit $P \cdot e_{n^{\prime} p}$ l'ensemble commun à $P$ et à $e_{n^{\prime} p}(\alpha)$. On a, d'après la remarque faite à l'instant:

$$
P=P \cdot e_{n^{\prime} 1}+P \cdot e_{n^{2} 2}+\ldots+P \cdot e_{n^{\prime} p}+\ldots,
$$

puisque tout point de $P$ appartient à un $e_{n^{\prime} p}(\alpha)$, done à un $P \cdot e_{x^{\prime} p_{0}}$ (et à tous les suivants, quand $p$ croît).

Il est donc impossible que tous les $P \cdot e_{n^{\prime},}$, soient non-denses sur l'ensemble parfait $P$. Car la réunion d'une infinité dénombrable d'ensembles situés' sur $P$ et non-denses sur $P$ ne peut constituer $P$.

Il y a done un nombre $p_{1}=\frac{1}{\eta_{1}}$ tel que l'ensemble $P \cdot e_{n \cdot p_{1}}=P \cdot e_{n}\left(H, f, \alpha, \eta_{1}\right)$ soit dense sur $P$, c'est-à-dire partout dense sur une portion $P^{\prime}$ de $P$. Mais alors $P^{\prime}$ est identique au dérivé de $P \cdot e_{n^{\prime} p}$, et par consóquent $P^{\prime}$ est a fortiori dans $\bar{e}_{n}\left(\alpha, \eta_{1}\right)$.

Par hypothèse $f_{n}(x, H)$ existe en tout point de $\bar{e}_{n}\left(H, f, \alpha, \eta_{1}\right)$. Done d'après le théorème III, en chaque point de $\bar{e}_{n}$ et spécialement à $\bar{e}_{n}, f_{n}(x, H)$ a une oscillation inférieure à $2 \alpha . \bar{e}_{n}$ contenant $P^{\prime}$, a fortiori en chaque point de $P^{\prime}$ et spécialement à $P^{\prime}$, l'oscillation de $f_{n}(x, H)$ est inférieure à $2 \alpha$. Done, sauf peut-Atre aux points extrêmes de $P^{\prime}$, l'oscillation de $f_{n}(x, H)$ spéciale à $P$ est, en tout point de $P^{\prime}$, au plus égale à $2 \alpha$, contrairement à l'hypothèse.

En résumé, $f_{n}(x, H)$ est ponctuellement discontinue sur tout ensemble parfait $P$ situé sur $H$. Done $f_{n}(x, H)$ est de classe 1 sur $H$.
19. Il semble difficile dans le cas d'un ensemble parfait quelconque $H$, d'obtenir un résultat plus précis que le théorème III.

Soit $\lambda_{1}=1, \lambda_{0}, \ldots, \lambda_{n}, \ldots, \lambda_{\infty}=0$ une suite de nombres non négatifs, tels que $\frac{\lambda_{n+1}}{\lambda_{n}}=\varepsilon_{n}<\frac{1}{8}$ tende vers 0 quand $n$ croît.

L'ensemble $H$ des points:

où

$$
x=\lambda_{t_{0}}-\lambda_{t_{1}}+\lambda_{t_{3}}-\ldots+(-1)^{n} \lambda_{t_{n}}+\ldots
$$

$$
0 \leqslant i_{0}<i_{1}<i_{2}<\ldots
$$

est parfait. Nous admettons $i_{n}=\infty$.

Sur $H$, considérons la fonction:

$$
f(x)=\sum(-1)^{n} \frac{\lambda_{i_{n}}^{3}}{6}
$$

$f(x)$ est continue sur $B$. Soient $x$ et $x^{\prime}$ deux points de $H$. Supposons que $x$ et $x^{\prime}$ débutent par les $n$ mêmes premiers chiffres:

$$
\lambda_{i_{0}}-\lambda_{i_{1}}+\ldots+(-1)^{n-1} \lambda_{i_{n-1}}=\sigma_{n-1}
$$

et que les $n^{\text {os }}$ chiffres diffèrent, soit $\lambda_{i_{n}} \neq \lambda_{i_{n}}$.

Si $i_{n}^{\prime}>i_{n}$, on a $x^{\prime}-x=(-1)^{n+1} \lambda_{i_{n}}\left(1+\delta_{n} \varepsilon_{n}\right)$ et $f\left(x^{\prime}\right)-f(x)$ $=(-1)^{n+1} \frac{\lambda_{i_{n}}^{3}}{6}-\left(1+\delta_{n}^{\prime} \varepsilon_{n}\right), \delta_{n}$ et $\delta_{n}^{\prime}$ étant bornés indépendamment de $n$.

Si $i_{n}>i_{n}^{\prime}$, on échange les indices $i_{n}$ et $i_{n}^{\prime}$ dans les relations précédentes et en même temps les signes des seconds membres.

Dans tous les cas

$$
f\left(x^{\prime}\right)-f(x)=\frac{\left(x^{\prime}-x\right)^{3}}{6}(1+\varepsilon)
$$

$\varepsilon=\varepsilon\left(x, x^{\prime}\right)$ tendant vers 0 avec $x^{\prime}-x$.

Donc, $f(x)$ possède spécialement à $B$ une différentielle troisième égale à $h^{3}$, si $h$ est l'accroissement de $x$. D'autre part,

$$
f_{1}(x, H)=0, \quad f_{2}(x, B)=0 \quad \text { avee } \quad f_{3}(x, H)=1 .
$$

$f_{1}(x, H)$ et $f_{2}(x, H)$ sont bien continues l'une et l'autre. Il en est encore de même de $f_{3}(x, H)$. Mais $f_{3}(x, H)$ n'est pas la dérivée de $f_{2}(x, H)$ spéciale à $H$, qui existe et est 0 , ni le second quotient différentiel de $f_{1}(x, H)$ spécial à $H$, qui existe et est 0 .

L'indice de $H$ est infini en chaque point, mais $\varepsilon_{n}$ tendant vers 0 
aussi lentement qu'on veut, l'ordre d'infinitude de l'indice de $H$ est aussi faible qu'on le veut. Oeci montre le rôle nécessaire joué par ce caractère de l'indice dans les propriétés de $H$ relativement à la différentiation.

20. Le fait que $f_{n}(x)$ [et même $f_{n}(x, H)$ ] soit une fonction de classe 1 quand elle existe (théorème IV) entraîne que, $P$ étant un ensemble parfait quelconque (situé sur $H$, si $f_{n}$ est spécial à l'ensemble parfait $H$ ), les points $\xi$ de $P^{2}$ au voisinage desquels le nombre $\varepsilon_{n}(x, h)$ des formules (1) [et (8)] est non borné indépendamment de $x$ et de $h$, forment eux mêmes un ensemble $K$ (fermé) non-dense sur $P$.

Si l'on remplace dans l'énoncé précédent le nombre $\varepsilon_{n}(x, h)$ par le nombre $\Phi_{n}(x, h)$ de la formule (9), la proposition reste exacte quand on suppose seulement que les quatre cocfficients differentiels $n^{\text {es }}$ extrêmes de $f$ (spéciaux à $H$, et au nombro do deux seulement aux points de première espèce do $H$ ) sont jinis en chaque point de $a b$ (de $H)$.

La démonstration du théorème IV justifie sans difficulté cette dernière extension.

De là la conséquence suivante:

21. Convenons de dire que, en un point $x$ de l'ensemble parfait $P, f(x)$ est régulière d'ordre $n$ spécialement $a ̀ ~ P$ si: $1^{0} f$ admet spécialement à $P$ une différentielle d'ordre $n-1$, avec les quotients successifs $f_{1}(x, P), \ldots, f_{n-1}(x, P)$ et tous les quatre (ou deux) coefficients différentiels extrêmes d'ordre $n$ finis; $2^{0}$ pour $p=1, \ldots, n-1$, $f_{p}(x, P)$ admet spécialement à $P$ une différentielle d'ordre $n-p-1$ avec les quotients spéciaux successifs $f_{p+1}(x, P), \ldots, f_{n-1}(x, P)$ et quatre coefficients différentiels extrêmes d'ordre $n$ fini.

Cela étant, nous avons le théorème suivant:

Théorème $\nabla$. Soit $f(x)$ défini sur ab (ou sur $H$ d'indice fini) et ayant en tout point ses coefficients différentiels extrêmes d'ordre $n$ (généraux ou spéciaux à $H$ ) finis. Quel que soit l'ensemble parfait $P$ situé sur $a b$ (ou sur $H$ ), l'ensemble $K_{0}$ des points $\xi_{0}$ de $P$ où, spécialement dे $P, f$ n'est pas régulière, est non-dense sur $P$.

Bien entendu, les quotients $f_{p}(x, P)$ sont alors identiques a $f_{p}(x)$ [ou à $f_{p}(x, H)$, puisque $P$ est dans $\left.H\right]$.

Les raisonnements donnés plus hauts et établissant ce théorème donnent même le résultat plus précis suivant:
21 b. Si $\xi$ désigne un point de $P$ tel que, pour tout intervalle $i$ de centre $\xi$, sur la portion $\omega=i \cdot P$ de $P$, les hypothèses du théorème II sont inexactes (pour tout choix de $\alpha$ et de $\eta$ positifs), l'ensemble $K$ des points analogues à $\xi$ est non-dense sur $P$.

Alors toute portion de $P$ sans point commun avec $K$ est une portion $P_{1}$ pour laquelle les hypothèses et les conclusions du théorème II sont vérifiées (pour certains nombres $\alpha, \eta$ dépendant de $P_{1}$ ).

Dans le cas oì $H$ est un ensemble parfait d'indice fini en chacun de ses points, il faut en outre observer que l'ensemble $K^{\prime}$ des points de $P$ oì l'indice de $H$ n'est pas uniformément fini, est non-dense sur $P$.

22. Enfin, si l'on suppose encore l'existence d'un quotient différentiel $n^{\theta}$ fini (général ou spécial à $H$ ) en tout point $x$ de $a b$. (ou de $H$ ), et non pas seulement la condition que tous les coefficients différentiels extrêmes $n^{\text {as }}$ soient finis, on voit que, sur toute portion $P_{1}$, l'ensemble des points oì $f_{p}(x)$ [ou $f_{p}(x, H)$ ] admet spécialement à $P_{1}$ une différentielle $(n-p)^{\text {e }}$ égale à $f_{n}(x)$ [ou à $f_{n}(x, H)$ ] est un résiduel de $P_{1}$.

Sous une forme plus brève, mais moins précise, nous pouvons énoncer le

Thérème VI. Si $f(x)$ admet en tout point de $a b$ (ou de l'ensemble parfait $H$ d'indice fini en chacun de ses points) une différentielle $n^{\circ}$ (générale ou spéciale à $H$ ), le système des $f_{p}(x)$ $(p=1,2, \ldots, p-1)$ est, sur tout ensemble parfait $P$ (inclus dans $B$ ), ponctuellement non différentiable à l'ordre $n-p$ spécialement à $P$.

Il faut entendre par là que toute portion de $P$ contient des point où chaque $f_{p}(x)$ est différentiable à l'ordre $n-p$ spécialement à $P$.

\section{Fonctions à coefficients différentiels $\boldsymbol{n}^{\text {es }}$ unilatéraux finis.}

23. Nous nous proposons de démontrer le théorème suivant:

Thérème VIT. $f(x)$ étant une fonction définie et continue sur un segment $a b$, si $E$ désigne l'ensemble des points de ab où, au moins d'un côté, $f(x)$ possède deux coefficients différentiels $n^{\text {es }}$ extrềmes finis, $f(x)$ admet une différentielle $n^{\mathrm{\theta}}$ générale sur une pleine épaisseur de $E$.

Pour mettre en défaut ce théorème, il faudrait qu'il fût possible de trouver une fonction $f(x)$ et un ensemble épais $I$ en tout point 
duquel, au moins d'un côté, $f(x)$ possédât deux coefficients différentiels $n^{\text {os }}$ extrêmes finis et distincts.

$I$ se décompose en deux ensembles $I_{1}$ et $I_{2}, I_{1}$ étant formé des points où la circonstance caractéristique de $I$ se présente au moins pour le côté droit, et $I_{2}=I-I_{1}$, où elle ne se présente pas pour le côté droit, donc nécessairement pour le côté gauehe.

L'un des deux ensembles $I_{1}$ et $I_{2}$ est épais, puisque $I=I_{1}+I_{2}$ l'est. On peut toujours, quitte à changer $x$ en $-x$, supposer que $I_{1}$ est épais.

Soit $f_{p}^{+}(x)(p=1,2, \ldots, n-1)$ le $p^{\theta}$ quotient différentiel droit de $f(x)$ au point $x$. Ecrivons:

$$
\begin{gathered}
f(x+h)=f(x)+h f_{1}^{+}(x)+\ldots+\frac{h^{p}}{p !} f_{p}^{+}(x)+\ldots \\
\ldots+\frac{h^{n-1}}{(n-1) !} f_{n-1}^{+}(x)+\frac{h^{n}}{n !} l_{n}(x, h) .
\end{gathered}
$$

Soit $A(x)$ la plus grande limite de $\left|l_{n}(x, h)\right|$ quand $h$ tend vers 0 par valeurs positives. Par hypothèse $A(x)$ est fini, quel que soit $x$ sur $I_{1}$. Done, si $\alpha>A(x)$, il existe un nombre positif $\eta$ tel que l'inégalité $0<h<\eta$ entraîne $\left|l_{n}(x, h)\right|<\alpha$. Mais $\alpha$ étant donné, $\eta$ dépend a priori non seulement de $\alpha$, mais anssi de $x$.

$r$ et $s$ étant deux entiers positifs quelconques, soit $I_{1 \cdot r \cdot s}$ l'ensemble des points $x$ de $I_{1}$ tels que, pour $0<h<1 / s$, on ait $\left|l_{n}(x, h)\right|<r$. Tout point $x$ de $I_{1}$ appartient à un ensemble $I_{1}$ r.rs (et dès lors à $I_{1 \cdot r, s}$ si $r^{\prime}>r$ et à $I_{1 \cdot r \cdot a^{\prime}}$ si $s^{\prime}>s$ ). Donc $I_{1}=\sum_{r, s} I_{1, r \cdot s}$. Les $I_{1, r s}$ sont en infinité dénombrable. Ils ne peuvent être tous minces (de mesure nulle), sans quoi $I_{1}$ serait lui aussi mince. Il existe done un nombre $\alpha=r_{0}$ et un nombre $\eta=1 / s_{0}$, tels que l'ensemble $I_{1}(\alpha, \eta)$ des points $x$ de $I_{1}$ vérifiant $\left|l_{n}(x, h)\right|<\alpha$ moyennant $0<h<\eta$, est épais. Dans cet ensemble $I_{1}(\alpha, \eta)$, nous pouvons prendre un ensemble parfait $Q$ épais (nous pouvons même supposer $Q$ épais en lui-même en retranchant les points autour desquels il est mince).

Le problème est réduit à montrer que, sur une certaine pleine épaisseur d'un ensemble parfait $Q$ oì $l_{n}(x, h)$ est uniformément borné par $\alpha$ (au sens que nous venons de dire), $f(x)$ possède une différentielle $n^{\circ}$ générale. Ceci résultera de l'extension suivante du théorème II aux hy-
pothèses actuelles:
24. Théorème VIII. Si $f(x)$ possède en tout point d'un ensemble parfait $P$ deux coefficients différentiels $n^{\text {es }}$ droits finis, de façon que l'égalité:

(18) $f(x+h)=f(x)+h f_{1}^{+}(x)+\ldots+\frac{h^{n-1}}{(n-1) !} f_{n-1}^{+}(x)+\delta a \frac{h^{n}}{h !}$

entraîne $|\delta|<1$ moyennant $0<h<\eta$, quel que soit $x$ sur $P, \alpha$ et $\eta$ étant indépendants de $x$ et $h$,

sous ces conditions suffisantes:

$1^{0} f_{n-1}^{+}(x)$ est continu sur $P$ spécialement à $P$, et vérifie sur $P$ la condition:

$$
\left|f_{n-1}^{+}(x+h)-f_{n-1}^{+}(x)\right|<\nu \alpha|h|
$$

$2^{0}$ spécialement d̀ $P$, en chaque point $x$ de $P$, et pour $p=1,2, \ldots, n-2$, a) $f_{p}^{+}(x)$ est continu, admet une différentielle d'ordre $n-p$ spéciale à $P$, le $m^{0}$ quotient différentiel $\left[f_{p}^{+}\right]_{m}(x, P)$ de $f_{p}^{+}(x)$ spécial à $P$ étant égal à $f_{p+m}^{+}(x)$ pour $m=1,2, \ldots, n-p-1$;

b) plus précisément, si $x$ et $x+k$ sont sur $P$, on a la formule:

$$
\begin{gathered}
f_{p}^{+}(x+k)=f_{p}^{+}(x)+k f_{p+1}^{+}(x)+\cdots \\
\cdots+\frac{k^{n-p-1}}{(n-p-1) !} f_{n-1}^{+}(x)+\delta \frac{k^{n-p}}{(n-p) !} \nu \alpha
\end{gathered}
$$

avec $\delta^{\mathrm{a}}<1$ si $|k|<\eta, \nu$ étant un certain coefficient indépendant de $x$, de $h$, de $\alpha$, et dépendant seulement de $n$.

La démonstration du théorème VIII est toute pareille à celle du théorème II pour les parties analogues des deux énoncés.

Dans la relation (4) faisons $A_{p}(y)=f_{p}^{+}(y)$ pour $p=1,2, \ldots, n-1$, $A_{n}(y)=0 . \quad x$ et $x+k$ étant deux points de $P$, la relation (4) est exacte pour $y=x$ et $y=x+k$, avec $y^{\prime}=x+k+h_{i}$, pourvu que $k+h_{i}$ et $h_{i}$ soient positifs et inférieurs à $\eta$.

Si $k>0$, on prendra $h_{i}=\left(\gamma_{i}-1\right) k, 1<\gamma_{1}<\gamma_{2}<\ldots<\gamma_{n+1}$. Si $k<0$, on prendra $h_{t}=\left(-\gamma_{t}-1\right) k$, les $\gamma_{i}$ étant indépendants de $k$ avec $\left(\gamma_{n+1}+1\right)|k|<\eta$.

Les formules (5) s'appliquent, $\mu_{p}$ ayant une certaine valeur indépendante de $x$ et de $k$, et inférieure à un nombre $\nu$ dépendant seulement des $\gamma_{i}$. Elles fournissent les égalités contenues dans l'énoncé du théorème VIII. 
25. Le théorème VIII n'exige rien concernant les propriétés métriques de l'ensemble parfait $P$. En supposant désormais $P$ épais, nous démontrerons le théorème VII, sous la forme réduite où nous avons amené son énoncé.

On conclut de $\left(19_{n-1}\right)$ que, sur pleine épaisseur de $P, f_{n-1}^{+}$a une dérivée unique spéciale à $P$. Soit $E_{0}$ l'ensemble des points de seconde espèce de $P$ où cette dérivée existe. Nous poserons $g_{n}(x)=$ $=\left[f_{n-1}^{+}\right]_{1}(x, P)$ sur $E_{0}, g_{n}(x)=0$ hors de $E_{0}$.

On a $\left|g_{n}(x)\right|<\nu a$, quel que soit $x$, entre les extrémités $c, d$ de $P$.

Soit $E_{1}$ l'ensemble des points de $E_{0}$ où $P$ a l'épaisseur 1 . $E_{1}$ est encore une pleine épaisseur de $P$.

Je dis qu'en tout point de $E_{1}, f(x)$ admet une différentielle $n^{\circ}$ gdnérale, le $n^{\circ}$ quotient différentiel de $f$ étant $g_{n}(x)$.

Nous montrerons auxiliairement que, en tout point $x$ de $E_{1}^{t}$, $f_{p}^{+}(x)(p=1,2, \ldots, n-1)$ admet, spécialement d̀ $P$, une différentielle d'ordre $(n-p)$, le quotient différentiel correspondant $6 \tan ^{t}$ $g_{n}(x)$; autrement dit:

$$
\begin{gathered}
f_{p}^{+}(x+k)=f_{p}^{+}(x)+k f_{p+1}^{+}(x)+\ldots \\
\cdots+\frac{k^{n-p-1}}{(n-p-1) !} f_{n-1}^{+}(x)+\frac{k^{n-p}}{(n-p) !} g_{n}(x)+o\left(k^{n-q}\right),
\end{gathered}
$$

en utilisant la notation $o(u)$ de $\mathrm{E}$. Landa u pour désigner un nombre dont le rapport à $u$ tend vers 0 .

Nous introduirons dans les raisonnements une légère simplification. Décomposons $P$ en un nombre fini de portions séparées par des intervalles contigus, le segment des points extrêmes de chaque portion étant inférieur à $\eta$ en longueur. Si le théorème est démontré pour chacune de ces portions, il est établi par là-même pour $P$. Supposons done que le segment $c d$ limité par les extrémités de $P$ soit inférieur à $\eta$. Alors, quels que soient $x$ et $x^{\prime}$ sur $P$,

$$
\left|f_{n-1}^{+}\left(x^{\prime}\right)-f_{n-1}^{+}(x)\right|<\nu \alpha\left|x^{\prime}-x\right| .
$$

26. Il nous sera utile de rappeler les notations commodes dont je me suis servi dans mon mémoire sur les nombres dérivés du premier ordre. Je désigne par $V\left(g, x, x^{\prime}\right)$ la variation ${ }_{n}^{\text {simple }}{ }^{\prime \prime}$ de $g$ entre $x$ et $x^{\prime}$, soit $g\left(x^{\prime}\right)-g(x)$; par $V R\left(g, x, x^{\prime}\right)$, la variation
${ }_{\text {nelative }}$ de $g$ entre $x$ et $x^{\prime}$, soit:

$$
\frac{g\left(x^{\prime}\right)-g(x)}{x^{\prime}-x}=\frac{V\left(g, x, x^{\prime}\right)}{x^{\prime}-x}
$$

Soient $a_{m} b_{n t}$ ou $u_{m}$ les contigus énumérés de $P$. D'après $u_{m}<\eta$, nous avons dans le cas qui nous occupe $\left|V R\left(f_{n-1}^{+}, u_{m}\right)\right|<v a u_{m}$. Soit $w_{m}$ un nombre attaché à $u_{m}$. Supposons que la série $\Sigma\left|w_{m}\right|$ converge. $x$ et $x^{\prime}$ appartenant au segment $c d$, nous désignons par $\left(x \Sigma x^{\prime}\right) w_{m}$

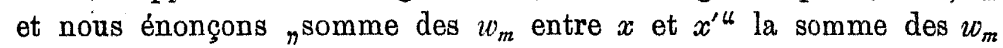
correspondant aux intervalles contigus $u_{m}$ totalement contenus dans l'intervalle $x x^{\prime}$. Ces $u_{m}$ vérifient les inégalités (si $\left.x<x^{\prime}\right) x \leqslant a_{m}<b_{m} \leqslant x^{\prime}$.

Si $x^{\prime}<x$, nous convenons de poser $\left(x \Sigma x^{\prime}\right) w_{m}=-\left(x^{\prime} \Sigma x\right) w_{m}$. Si l'épaisseur moyenne de $P$ sur $x x^{\prime}$ est supérieure à $1-\varepsilon$, on a évidemment $\left|\left(x \Sigma x^{\prime}\right) u_{m}^{r}\right|<\varepsilon^{r}\left|x^{\prime}-x\right|^{r}$.

Si l'épaisseur de $P$ au point $x$ est 1 , le nombre $\left|\left(x \Sigma x^{\prime}\right) u_{m}^{r}\right|+$ $+\theta u_{q}^{r}$ vaut $o\left[\left(x^{\prime}-x\right)^{r}\right]$ quand $x^{\prime}$ tend vers $x$, si l'on fait $\theta=0$ quand $x^{\prime}$ est sur $P, \theta=1$ si $x^{\prime}$ est dans l'intervalle $u_{q}$. Cette remarque évidente nous servira plusieurs fois.

27. Ces notations étant posées, nous considérerons une suite de fonctions $g_{n-1}(x), g_{n-2}(x), \ldots, g_{1}(x), g(x)$, toutes définies et continues sur le segment $c d$, et déterminées par les conditions suivantes:

a) Si $x$ est situé sur $P, g_{p}(x)=f_{p}^{+}(x)$.

b) Si $x$ est un point quelconque de $c d$, la fonction $\varphi_{p}(x)$ definie par:

$$
g_{p}(x)=f_{p}^{+}(c)+\int_{c}^{x} g_{p+1}(x) d x+\varphi_{p}(x)
$$

est linéaire sur chaque contigu d̀ $P$ (et continue sur $c d$, d'après l'hypothèse que $g_{p}(x)$ est continu).

Ces conditions suffisent à définir de proche en proche toutes les fonctions $g_{p}(x)$, pour $p \leqslant n-1$. Sur l'intervalle $a_{m} b_{m}$ contigu à $P$, nous poserons:

$$
\varphi_{p}(x)-\varphi_{p}\left(a_{m}\right)=\lambda_{p, m}\left(x-a_{m}\right) .
$$

Nous introduirons encore une fonction auxiliaire $R_{p}(x, h)$ par l'égalité:

$$
\begin{aligned}
& g_{p}(x+h)=g_{p}(x)+h g_{p+1}(x)+\ldots \\
& \ldots+\frac{h^{n-p-1}}{(n-p-1) !} g_{n-1}(x)+R_{p}(x, h) .
\end{aligned}
$$

Nous nous proposons d'établir les propriétés suivantes de $\varphi_{p}(x)$ et de $R_{p}(x, h)$ : 
28. A 1. $\varphi_{p}(x)$ vérifie sur cd une condition de Lipschitz, et par suite est l'intégrale indéfinie de sa dérivée (remplacée par 0 aux points de l'ensemble mince où $\varphi_{p}^{\prime}(x)$ n'existe pas).

Cette propriété de $\varphi_{p}(x)$ résulte de la remarque que voici:

Quand une fonction $\varphi(x)$ linéaire sur les contigus à l'ensemble parfait $\mathrm{P}$ d'extrémités $c, d$ vérifie l'inégalité:

$$
\left|\varphi\left(x^{\prime}\right)-\varphi(x)\right|<K\left|x^{\prime}-x\right|,
$$

quels que soient $x$ et $x^{\prime}$ situées simultanément sur $P$, elle vérifie la même condition, quels que soient $x$ et $x^{\prime}$ sur $c d$ ( $K$ indépendant de $n$ et de $x^{\prime}$.

La démonstration est immédiate, si l'on remarque que sur chaque contigu $a_{m} b_{m}$ dे $P$, on a $\left|V R\left[\varphi, a_{m}, x\right]\right|=\left|V R\left(\varphi, a_{m}, b_{m}\right)\right|<K$. D'après une proposition fondamentale due à H. Lebesgue, $P(x)$ admet sur une pleine épaisseur de $P$ une dérivée finie $\varphi^{\prime}(x)$. Hors de $P,\left|\varphi^{\prime}(x)\right|=\left|\nabla R\left(\varphi, a_{m}, b_{m}\right)\right|<K$. Sur une pleine épaisseur de $P$, $\varphi^{\prime}(x)$ existe et $\mathrm{y}$ vérifie $\left|\varphi^{\prime}(x)\right| \leqslant K$.

Appliquons ces remarques à $\varphi_{p}(x)$. La fonction $g_{p}(x)$ coïncide sur $P$ avec $f_{p}^{+}(x)$ qui, d'après la relation $\left(19_{p}\right)$, vérifie la condition de Lipschitz entre deux points quelconques $x, x^{\prime}=x+k$ de $P$. La fonction $\int_{c}^{x} g_{p+1}(x) d x$ vérifie également une condition de Lipschitz sur $c d$, puisque sa dérivée $g_{p+1}(x)$ est bornée sur tout $c d$. Done, $\varphi_{p}(x)$ vérifie une condition de Lipschitz entre deux points quelconques $x, x+k$ de $P$ et par suite aussi sur la totalité du segment $c d$.

Nous tirons de là cette conséquence que $g_{p}(x)$ donné par (21) vérifie lui aussi une condition de Lipschitz sur la totalité du segment $c d$.

A 2. $\varphi_{p}^{\prime}(x)=0$ sur une pleine épaisseur de $P$.

En effet, sur une pleine épaisseur de $c d, g_{p}(x)$ qui vérifie une condition de Lipschitz a une dérivée bornée sur une épaisseur pleine, en particulier sur une pleine épaisseur $E_{2}$ de $P$. Mais, sur $P, g_{p}(x)$ coìncide avec $f_{p}^{+}(x)$. Si donc en un point de $P, g_{p}(x)$ a une dérivée (générale), cette dérivée coïncide avec la dérivée de $f_{p}^{+}(x)$ spéciale à $P$, donc d'après $\left(19_{p}\right)$ avec $f_{p+1}^{+}(x)=g_{p+1}(x)$, D'après l'expression $\left(21_{p}\right)$ de $g_{p}(x)$, en tout point de $E_{2}, \varphi_{p}^{\prime}(x)$ existe et vaut 0.

Or, $\varphi_{p}(x)$ qui vérifie une condition de Lipschitz sur $c d$ a une dérivée bornée sur une pleine épaisseur de $c d$, et si on remplace $\varphi_{p}^{\prime}(x)$ par 0 aux points de l'ensemble mince où $\varphi_{p}^{\prime}(x)$ n'existe pas, on a

$$
\varphi_{p}(x)=\int_{c}^{x} \varphi_{p}^{\prime}(x) d x .
$$

Mais, sur $P, \varphi_{p}^{\prime}=0$. Sur $u_{m}, \int_{a_{m}}^{x} \varphi_{p}^{\prime}(x) d x=\lambda_{p \cdot m}\left(x-a_{m}\right)$. Finalement:

$$
\varphi_{p}(x)=(c \Sigma x) \lambda_{p \cdot m} u_{m}+\theta \lambda_{p \cdot q}\left(x-a_{q}\right),
$$

avec $\theta=0$ si $x$ est sur $P$ et $\theta=1$ si $x$ est sur l'intervalle $u_{q}$.

La formule $\left(24_{p}\right)$ montre en même temps qu'en tout point $x$ de $P$ où l'épaisseur de $P$ est $1, \varphi_{p}^{\prime}(x)=0$. Il en est done ainsi en particulier sur $E_{1}$.

29. B. Evaluons maintenant $R_{p}(x, h)$. Nous allons établir les formules suivantes:

$$
\left|R_{p}(x, k)\right|<\nu \alpha \frac{|k|^{n-p}}{(n-p) !}
$$

$(x \operatorname{sur} P, x+k \operatorname{sur} P, p=0,1, \ldots, n-1)$.

Ceci résulte des formules $(18)$ et $\left(19_{p}\right)$ et de l'égalité $g_{r}(x)=f_{r}^{+}(x)$ quand $x$ est sur $P$

$$
\left|R_{p}(x, h)\right|<\nu \alpha\left[\frac{|h|^{n-p}}{(n-p) !}+\gamma_{p}|h| u_{q}^{n-p-1}\right]
$$

$\left(x \operatorname{sur} P, x+h\right.$ sur $\left.\boldsymbol{u}_{q}\right), \gamma_{p}$ ne dépendant que de $p$ (et de $n$ ) mais non pas de $x$ ni de $h$.

$$
R_{p}(x, h)=\frac{h^{n-p}}{(n-p) !} g_{n}(x)+o\left(h^{n-p}\right)
$$

$\left(x \operatorname{sur} E_{1}, x+h \operatorname{sur} c d\right)$.

Il résultera de (25 ter) que, aux points de $E_{1}, g_{p}(x)$ a sa dérivée $(n-p)^{\bullet}$ égale à $g_{n}(x)$. Cette propriété pour $p=0$ nous permettra d'établir immédiatement le théorème que $g_{n}(x)$ est le $n^{\mathrm{e}}$ quotient différentiel ordinaire de $f(x)$ en tout point de $E_{1}$.

Etablissons d'abord les formules (25 bis), (25 ter) pour $p=n-1$. Nous avons

$$
g_{n-1}(x)=f_{n-1}^{+}(c)+\int_{c}^{x} g_{n}(x)+\varphi_{n-1}(x)
$$

et nous posons

$$
g_{n-1}(x+h)=g_{n-1}(x)+R_{n-1}(x, h)
$$


$g_{n-1}(x)$, égal à $f_{n-I}^{+}(x)$ sur $P, \mathrm{y}$ vérifie l'inégalité

$$
\left|g_{n-1}\left(x^{\prime}\right)-g_{n-1}(x)\right|<\nu \alpha\left|x^{\prime}-x\right|
$$

quels que soient $x$ et $x^{\prime}$ sur $P$.

$g_{n-1}$, linéaire sur les contigus à $P$, puisque $g_{n}(x)=0$ sur chaque segment $a_{m} b_{m}$, vérifie la même inégalité, quels que soient $x$ et $x^{\prime}$ sur $c d$. Donc,

$$
\left|R_{n-1}(x, g)\right|<\nu \alpha|h|
$$

ce qui est la formule (25 bis) axec $\gamma_{n-1}=0$.

En un point de $E_{1}$ (et même de $E_{0}$ ), $g_{n-1}^{\prime}$ a pour dérivée générale la dérivée de $f_{n-1}^{+-1}(x)$ spéciale à $P$, soit $g_{n}(x)$. Done

$$
g_{n-1}(x+h)-g_{n-1}(x)=h g_{n}(x)+o(h)
$$

$(x$ sur $E, x+h$ sur $c d)$. D'où $R_{n-1}(x, h)=h g_{n}(x)+o(h)$ si $x$ est. sur $E_{1}$ (meme sur $E_{0}$ ). Les formules (25 bis) et (25 ter) sont donc établies pour $p=n-1$.

$29 \mathrm{~b}$. Supposons démontrées les formules (25 bis) et $(25$ ter) pour tous les indices supérieurs à $p$, donc pour $g_{n-1}, g_{n-2}, \ldots, g_{p+1}$ et prouvons-les pour $g_{p}(x)$.

Comparons les deux expressions de $g_{p}(x+h)-g_{p}(x)$ fournies par la formule (23) et par la relation (21) définissant $g_{p}(x)$. Celle-ci donne

$$
g_{p}(x+h)-g_{p}(x)=\int_{x}^{x+h} g_{p+1}(t) d t+\varphi_{p}(x+h)-\varphi_{p}(x) .
$$

L'intégrale vaut $\int_{0}^{h} g_{p+1}(x+u) d u$, et d'après la formule (23) admise quand on y remplace $p$ par $p+1$, un calcul immédiat donne

$$
\begin{aligned}
& \int_{x}^{x+h} g_{p+1}(t) d t=h g_{p+1}(x)+\frac{h^{2}}{2} g_{p+2}(x)+\ldots \\
& \ldots+\frac{h^{n-p-2}}{(n-p-2) !} g_{n-1}(x)+\int_{0}^{h} R_{p+1}(x, u) d u .
\end{aligned}
$$

Finalement

$$
R_{p}(x ; h)=\int_{0}^{h} R_{p+1}(x, u) d u+\varphi_{p}(x+h)-\varphi_{p}(x) .
$$

Cette formule est établie, quels que soient $x$ et $x+h$ sur $c d$. Faisons $x=a_{m}, x+h=b_{m}$, d'où $h=u_{m}$. D'après $\varphi_{p}\left(b_{m}\right)-\varphi_{p}\left(a_{m}\right)=\lambda_{p \cdot m} u_{m}$

\section{on a}

$$
\lambda_{p \cdot m} u_{m}=R_{p}\left(a_{m}, u_{m}\right)-\int_{0}^{u_{m}} R_{p+1}\left(a_{m}, u\right) d u \text {. }
$$

Mais $a_{m}$ et $b_{m}$ étant sur $P$, d'après la formule (25), établie quel que soit $p \leqslant n-1,\left|R_{p}\left(a_{m}, u_{m}\right)\right|<\nu \alpha \frac{u_{m}^{n-p}}{(n-p) !}$. D'autre part, d'après (25 bis) admis pour l'indice $p+1$,

\section{Finalement}

$$
\left|R_{p+1}\left(a_{m}, u\right)\right|<\nu a\left[\frac{u_{m}^{n-p-1}}{(n-p-1) !}+\gamma_{p+1} u \cdot u_{m}^{n-p-2}\right] .
$$

en posant

$$
\left|\lambda_{p^{\prime} m}\right|<\mu_{p} \nu \alpha u_{m}^{n-p-1}
$$

$$
\mu_{p}=\frac{2}{(n-p) !}+\frac{\gamma_{p+1}}{2} \text {. }
$$

On en conclut immédiatement que si $x$ est sur $P$ et $x+h$ dans $u_{q}$,

$\left|\varphi_{p}(x+h)-\varphi_{p}(x)\right|<\mu_{p} \nu \alpha\left|(x \Sigma x+h) u_{m}^{n-p}\right|+\mu_{p} \nu \alpha u_{q}^{n-p-1}|h|$,

et par suite quand $x$ est un point de $P$ où l'épaisseur de $P$ est 1 , (29 ter)

$$
\varphi_{p}(x+h)-\varphi_{p}(x)=o\left(h^{n-p}\right) .
$$

Nous n'écrirons pas les formules (29) et (29 bis), donnant respectivement $\varphi_{p}(x+h)-\varphi_{p}(x)$ pour $x$ et $x+h$ sur $P$ ou quelconques sur $c d$.

29 c. Le dernier terme de la formule (26) étant évalué, nous pouvons obtenir une limite supérieure de $\left|R_{p}(x, h)\right|$ dans le cas qui nous intéresse ( $x$ sur $P, x+h$ dans $u_{q}$ ).

$x+h$ étant dans $u_{q}$, on a d'après (26)

$$
\frac{d}{d h} R_{p}(x, h)=R_{p+1}(x, h)+\lambda_{p^{\prime} q} .
$$

D'après l'expression (25 bis). de $R_{p+1}(x, h)$ et la formule (28),

$$
\begin{aligned}
& \left|\frac{d}{d h} R_{p}(x, h)\right|<\nu \alpha\left[\frac{\mid h^{n-p-1}}{(n-p-1) !}+\gamma_{p+1}|h| u_{q}^{n-p-2}\right]+\mu_{q} \nu \alpha u_{q}^{n-p-1} . \\
& \text { Si } h>0, \quad R_{p}(x, h)=R\left(x, a_{q}-x\right)+\int_{a_{q-x}}^{h} \frac{d}{d u} R_{p}(x, u) d u .
\end{aligned}
$$


Mais, $a_{q}$ étant sur $P$, la formule (25) s'applique:

Donc

$$
\left|R_{p}\left(x, a_{q}-x\right)\right|<\nu \alpha \frac{\left(a_{q}-x\right)^{n-p}}{(n-p) !} .
$$

$$
\left|R_{p}(x, h)\right|<\nu \alpha \frac{h^{n-p}}{(n-p) !}+\left(\gamma_{p+}+\mu_{p}\right) \nu \alpha h u^{n-p-1},
$$

Si $h<0, R_{p}(x, h)=R\left(x, b_{p}-x\right)-\int_{h}^{b^{q^{-x}}} \frac{d}{d \dot{u}} R_{p}(x, u) d u$.

Done, quel que soit $x+h$ sur $u_{q}$, la formule (25 bis) est établio avec $\gamma_{p}=\gamma_{p+1}+\mu_{p}$.

Quant à la formule (25 ter) relative au cas où $x$ est sur $E_{1}$, elle résulte immédiatement $1^{n}$ de la relation (25 ter) admise pour l'indice $p+1$,

$$
R_{p+1}(x, h)=g_{n}(x) \frac{h^{n-p-1}}{(n-p-1) !}+o\left(h^{n-p-1}\right) ;
$$

$2^{0}$ de la relation (29 ter), valable parce que, en tout point de $E_{1}$, l'épaisseur de $P$ est 1 .

La formule (25 ter), qui nous intéresse particulièrement, est donc établie pour toute valeur de $p$. Il n'y a aucune difficulté complémentaire pour $p=0$.

30. On a donc, en remplaçant dans (23) $g_{r}(x)$ par $f_{r}^{+}(x)$ pour $r=0,1,2, \ldots, n-1$ et en supposant $x$ sur $E_{1}$ et $h$ quelconque sur $c d$ :

$$
\begin{gathered}
g(x+h)=f(x)+h f_{I}^{+}(x)+\ldots \\
\cdots+\frac{h^{n-1}}{(n-1) !} f_{n-}^{+}(x)+\frac{h^{n}}{n !} g_{n}(x)+o\left(h^{n}\right) .
\end{gathered}
$$

Il s'agit de borner $g(x+h)-f(x+h)=D(x, h)$

Si $x+h$ est sur $P, D(x, h)=0$.

Si $x+h$ est dans $u_{q}$, nous avons, d'après les formules (18) et (23) appliquées au point $a_{q}$,

$$
\begin{gathered}
f(x+h)=f\left(a_{q}\right)+\left(x+h-a_{q}\right) f_{1}^{+}\left(a_{q}\right)+\ldots \\
\ldots+\frac{\left(x+h-a_{q}\right)^{n-}}{(n-1) !} f_{n-1}^{+}\left(a_{q}\right)+\nu \alpha \frac{\left(x+h-a_{q}\right)^{n}}{n !} \quad\left(\delta^{2}<1\right), \\
g(x+h)=g\left(a_{q}\right)+\left(x+h-a_{q}\right) g_{1}\left(a_{q}\right)+\ldots \\
\ldots+\frac{\left(x+h-a_{q}\right)^{n-1}}{(n-1) !} g_{n-1}^{\cdot}\left(a_{q}\right)+R_{0}\left(a_{q}, x+h-a_{q}\right)
\end{gathered}
$$

avec $\left|R_{0}\left(a_{q}, x+h-a_{q}\right)\right|<\alpha \nu\left[\frac{\left(x+h-a_{p}\right)^{n}}{n !}+\gamma_{0}\left(x+h-a_{q}\right) u_{q}^{n-1}\right]$.

Done

$$
|g(x+h)-f(x+h)|<\nu \alpha\left(\frac{2}{n !}+\gamma_{0}\right) u_{q}^{n}
$$

si $x+h$ est dans $u_{q}$.

Si l'épaisseur de $P$ au point $x$ est $1, \frac{u_{q}}{h}$ tend vers 0 . Donc

$$
g(x+h)-f(x+h)=o\left(h^{n}\right)
$$

Finalement, en tout point $x$ de $E_{1}$, la formule (30) où l'on remplace $g(x+h)$ par $f(x+h)$ est justifiée, quel que soit $x+h$ sur $c d$.

Donc, $f(x)$ admet en tout point de $E_{1}$ une différentielle $n^{\mathrm{e}}$ ordinaire, $f_{p}^{+}(x)$ doit s'écrire $f_{p}(x)$ et $g_{n}(x)$ doit s'écrire $f_{n}(x)$, si $x$ est $\operatorname{sur} E_{1}$.

Le théorème VII est donc établi.

31. Il serait curieux de rechercher si les trois autres cas fondamentaux des nombres dérivés du premier ordre (voir mon mémoire du Journal de Math. pures et appliquées, 1915) sont également les seuls que les coefficients différentiels $n^{\text {es }}$ extrêmes d'une fonction $f(x)$ sont susceptibles de présenter sur un ensemble épais.

32. S'il s'agit de la différentiation spécialement à un ensemble parfait $H$ d'indice fini, les circonstances sont les mêmes que si $H$ est le continu. Le théorème VIII subsiste pour tout ensemble parfait inclus dans $H$ et sur lequel l'indice de $H$ est uniformément fini. Bien entendu, l'égalité (18), donnée dans l'énoncé du théorème VIII sous sa nouvelle forme, est subordonnée à l'hypothèse complémentaire que $x+h$ soit sur $H$.

Le théorème VIII jouera un rôle essentiel par la suite, grâce à cette remarque fondée sur un raisonnement analogue à ceux de Baire, ou de nature ${ }_{n}$ deseriptive ${ }^{u}$ :

Si en tout point d'un ensemble parfait $P, f$ possède $d u$ côté droit (ou toujours du côté gauche) deux quotients différentiels $n^{\text {es }}$ extrêmes finis,

ou bien il existe deux nombres positifs $\alpha$ et $\eta$ tels que l'égalité (18) ait lieu, quel que soit $x$ sur $P$ et $0<h<\eta$, 
ou bien il existe sur $P$ un ensemble fermé non-dense $K$ tel que sur toute portion de $P$ sans point commun avec $K$ la première alternative est réalisée.

32b. Supposons qu'en chaque point de $P$, l'existence de deux quotients différentiels $n^{\text {es }}$ extrêmes unilatéraux de $f$ soit assurée pour au moins un côté, sans que ce côté soit nécessairement toujours le même. Si $e_{n}^{+}(f, \alpha, \eta), e_{n}^{-}(f, \alpha, \eta)$ sont les ensembles des points $x$ où la condition (17) et la condition (17-) qu'on déduit de (17) en changeant $f_{p}^{+}$en $f_{p}^{-}$sont respectivement vérifiées avec $\left|l_{n}(x, h)\right| \leqslant \alpha$ pour $0<h<\eta$ ou pour $-\eta<h<0$, il existe des nombres positifs $\alpha, \eta$ tels que l'un au moins des deux ensembles $e_{n}^{+}$ou $e_{n}^{-}$soit partout dense sur une portion $P_{1}$ de $P$. Le raisonnement du théorème III montre que cet ensemble, $e_{n}^{+}$ou $e_{n}^{-}$, contient la totalité de $P_{1}$.

On en conclut que, quel que soit $P$, ou bien il existe deux nombres positifs $\alpha, \eta$ tels que l'une au moins des denix égalités (18) ou (18-) ait lieu quel que soit $x$ sur $P$, et respectivement $0<h<\eta$ ou $-\eta<h<0$.

Ou bien il existe sur $P$ un ensemble fermé $K_{1}$ non-dense sur $P$ et tel que, sur toute portion de $P$ sans point commun avec $K_{1}$, la première alternative se réalise.

\section{Quotients différentiels généralisés.}

33. Soit $f(x)$ une fonction définie sur le segment $a b$ et $\alpha_{1}, \alpha_{2}, \ldots, \alpha_{n+1}$ $(n+1)$ nombres indépendanțs de $x$ et de $h$. Posons

$$
\varphi(u)=\varphi\left(u, \alpha_{1}, \alpha_{2}, \ldots, \alpha_{n+1}\right)=\left(u-\alpha_{1}\right) \ldots\left(u-\alpha_{n+1}\right) .
$$

Nous appellerons combinaison aux différences de $f(x)$, définie par la suite $\alpha_{i}$, le nombre

$$
Q_{n}\left(f, x, h, \alpha_{1}, \ldots, \alpha_{n+1}\right)=n ! \sum_{i=1}^{i=n+1} \frac{f\left(x+\alpha_{i} h\right)}{\varphi^{\prime}\left(\alpha_{i}\right)} .
$$

L'addition à $f(x)$ d'un polynome quelconque de degré $n-1$ au plus en $x$ ne change pas $Q_{n}$.

Considérons le rapport

$$
R_{n}\left(f, x, h, \alpha_{1}, \ldots, \alpha_{n+1}\right)=\frac{Q_{n}\left(f, x, h, \alpha_{1}, \ldots, \alpha_{n+1}\right)}{h^{n}}
$$

Si $f(x)$ possède au point $x$ un quotient différentiel général égal à $f_{n}(x)$, la condition (1) et la formule (16) donnent:

$$
\begin{aligned}
& \text { (16 bis) } \quad f_{n}(x)=R_{n}\left(f, x, h, \alpha_{i}\right)-\sum_{i=1}^{i=n+1} \frac{\alpha_{i}^{n}}{\varphi^{\prime}\left(\alpha_{i}\right)} \varepsilon\left(x, \alpha_{i} h\right) \\
& \text { et } f_{n}(x)=\lim _{h=0} R_{n}\left(f, x, h, \alpha_{i}\right) \text {. }
\end{aligned}
$$

Nous dirons que $g_{n}(x)=g_{n}\left(x, a_{1}, \ldots, \alpha_{n+1}\right)$ est un quotient differentiel généralisé de $f$ au point $x$ si

(32) $\quad g_{n}(x)=\lim _{h=0} R_{n}\left(f, x, h, \alpha_{i}\right)=\lim _{h=0} \frac{n !}{h^{n}} \sum_{i=1}^{i=n+1} \frac{f\left(x+\alpha_{i} h\right)}{\varphi^{\prime}\left(\alpha_{i}\right)}$,

L'existence de $g_{n}(x)$ n'entraîne nullement l'existence du quotient différentiel ordinaire $f_{n}(x)$.

34. Comme dans le cas du quotient différentiel ordinaire $f_{n}(x)$, il est naturel de se poser les deux questions suivantes:

$1^{0}$ La connaissance de $g_{n}(x)$ en tout point de $a b$ pour une suite $\alpha_{\ell}$ donnée détermine-t-elle $f(x)$, à l'addition près d'un polynome quelconque de degré $n-1$ au plus?

$2^{0}$ Comment est-il possible de passer de $g_{n}(x)$ supposé donné à $f(x)$ inconnu?

Dans le cas où $n=2, \alpha_{1}=1, \alpha_{2}=0, \alpha_{3}=-1, g_{n}(x)$ est la dérivée seconde généralisée de $f(x)$, au sens de Riemann. On sait, d'après Schwartz, que la connaissance de $g_{2}(x)$ détermine $f(x)$ à l'addition près d'une fonction linéaire, et $j^{\prime}$ 'ai défini ${ }^{1}$ ) l'opération intégrale permettant d'obtenir $f$ au moyen de $g_{2}$.

Je n'aborderai ici ni la première question dans toute sa généralité [détermination de $f(x)$ par $g_{n}(x)$ ], ni, dans le cas de la détermination de $f(x)$ par $g_{n}(x)$, le problème d'intégration permettant d'obtenir $f$ au moyen de $g_{n}$.

35. Observons seulement que, si les $\alpha_{i}$ ne présentent pas les deux signes, $f(x)$ ne peut pas être déterminé par $g_{n}(x)$. Généralement, un quotient différentiel ordinaire unilatéral d'ordre au moins égal à 2 ne définit pas la fonction d'où il dérive.

Nous le montrerons a fortiori, en donnant l'exemple d'une fonction admettant en tont point et de chaque côté une différentielle unila-

1) C. R. Paris (t. 172, pp. $653,833,903,1218$; t. 173, p. 127), notes róunies en un fuscicule (Gauthier-Villars, Paris). Voir également mes deux Notices ayant pour objet l'exposé de mes travaux (1921 et 1934).

Fundamenta Mathematicae, T. XXV. 
térale $n^{\theta}$ nulle, sans que cette fonction soit identique da un polynome unique de degré $(n-1)$ au plus. Si même $E$ est un ensemble fermé indifféremment donné d'avance, on pourra faire en sorte que cette fonction ne soit identique à un polynome unique dans aucun intervalle contenant au moins un point de $E$.

Soit $u(x)=[d(x, E)]^{n+\alpha}(0<\alpha)$. Sur un intervalle contigu quelconque $i$ de $E$, d'extrémités $c, d$, plaçons une suite croissante $a_{m}$ $m$ allant de $-\infty$ à $+\infty, a_{m}$ tendant vers $c$ pour $m=-\infty$ et vers $d$ pour $m=+\infty$.

Donnons-nous indifféremment $f\left(a_{m}\right)$ non nul et inférieur en valeur absolue à $u\left(a_{m}\right)$ et, pour $a_{m} \leqslant x \leqslant a_{m-1-1}$, soit $f(x)=P(x, c d, m)$, $P$ étant un polynome de degré $n-1$, prenant aux extrémités du segment $a_{m} a_{m+1}$ les valeurs choisies $f^{\prime}\left(a_{m}\right), f^{\prime}\left(a_{m-1-1}\right)$ et vérifiant on outre l'inégalité $|P(x, c d, m)|<u(x)$ pour $a_{m}<x<a_{m+1}$.

Sur chaque contigu à $E$, procédons de même. Finalement $f(x)$ est défini sur la totalité de $a b$. Faisons $f(x)=0$ pour $x<a$ et pour $x>b . f(x)$ est continu sur tout l'axe réel.

En chaque point de $E, f$ a une différentielle ordinaire $n^{\theta}$ nulle, tous les $(n-1)$ premiers quotients différentiels étant également nuls.

En chaque point $a_{n}, f$ a de chaque côté une différentielle $n^{\ominus}$, le $n^{\ominus}$ quotient différentiel unilatéral étant 0 pour la droite et pour la gauche. Mais en général les quotients différentiels antérieurs au $n^{\theta}$ ne seront pas les mêmes au point $a_{m}$ pour le côté gauche et pour le côté droit.

Enfin, sur tout intervalle ne contenant aucun point $a_{m}, f(x)$ est un polynome de degré $n-1$, il a donc une dérivée ordinaire $n^{\theta}$ nulle.

Sur l'intervalle total $c d, f(x)$ n'est pas un polynome unique. Car ce polynome devrait, comme sur $a_{m} a_{m+1}$, être de degré $n-1$. Il s'annulerait $n$ fois en $c$ (et aussi en $d$ ). Il serait donc identiquement nul. Or, ceci est impossible d'après l'hypothèse $f\left(\alpha_{m}\right) \neq 0$.

$f(x)$ a donc toutes les propriétés annoncées.

36. Soient $x_{1}, x_{2}, \ldots, x_{n+1} n+1$ points quelconques situés sur $a b$. Posons $\psi\left(x, x_{1}, \ldots, x_{n+1}\right)=\psi(x)=\left(x-x_{1}\right)\left(x-x_{2}\right) \ldots\left(x-x_{n+1}\right)$ et

$$
V\left(f, x_{1}, x_{2}, \ldots, x_{n+1}\right)=V=\sum_{r=1}^{r=n+1} \frac{f\left(x_{r}\right)}{\psi^{\prime}\left(x_{r}\right)} .
$$

Si $g(x)$ est continue et est la dérivée $n^{\theta}$ d'une fonction $f(x)$, on trouve immédiatement, $\xi$ étant un nombre quelconque de $a b$;
(33) $\quad V=\frac{1}{(n-1) !} \sum_{r=1}^{r=n+1} \frac{1}{\psi^{\prime}\left(x_{r}\right)} \int_{\xi}^{x_{r}}\left(x_{r}-x\right)^{n-1} g(x) d x$.

On intègre $n$ fois par parties chacun des $n+1$ termes du second membre de $(33)$ et on utilise les égalités $\sum_{r=1}^{r=n+1} \frac{x_{r}^{p}}{\psi^{\prime}\left(x_{r}\right)}=0$, pour
$0 \leqslant p \leqslant n-1$. Posons

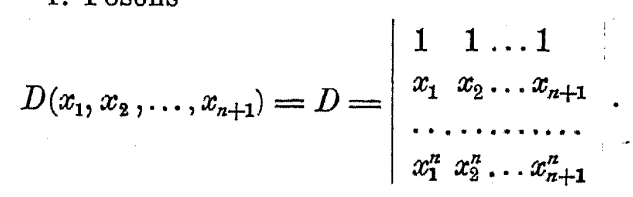

Soit $D_{r}$ le mineur de $x_{r}^{n}$ dans $D$, done

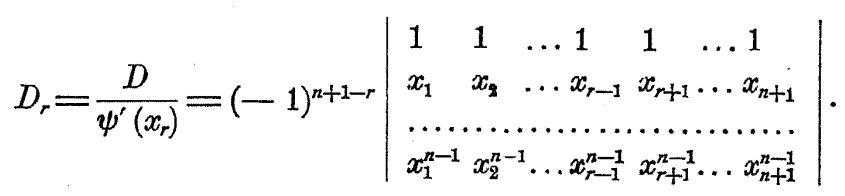

En multipliant les deux membres de (33) par $D$, on a

(34) $\quad U=\Sigma D_{r} f\left(x_{r}\right)=\frac{1}{(n-1) !} \sum_{r=1}^{r-n+1} D_{r} \int_{\xi}^{x_{r}}\left(x_{r}-x\right)^{n-1} g(x) d x$.

Le second membre, qui s'annule quand deux quelconques des $n+1$ quantités $x_{r}$ deviennent égales, est une sorte d'intégrale à $n+1$ limites. Son expression au moyen de la primitive $n^{\ominus}, f(x)$, est indépendante de l'addition à celle-ci d'un polynome arbitraire de degré $n-1$.

J'ai considéré, pour $n=2$, l'intégrale à trois limites

$$
T(g, a, b, c)=(c-b) f(a)+(a-c) f(c)+(b-a) f(c)
$$

qui, lorsque $g$ est contiuu (ou plus généralement sommable ou totalisable), s'exprime par une somme de trois intégrales simples.

Enfin, dans les égalités (33) ou (34) remplaçons $x_{n+1}$ par $x$. Posons $\quad P(x)=\left(x-x_{1}\right) \ldots\left(x-x_{n}\right)$, d'où $\quad \psi^{\prime}\left(x_{n+1}\right)=P(x)$, $\psi^{\prime}\left(x_{r}\right)=-P^{\prime}\left(x_{r}\right)\left(x-x_{r}\right)$. On obtient

$$
\begin{gathered}
f(x)-P(x) \sum_{r=1}^{r=n} \frac{f\left(x_{r}\right)}{P^{\prime}\left(x_{r}\right)\left(x-x_{r}\right)}=\frac{1}{(n-1) !} \int_{\xi}^{x}(x-t)^{n-1} g(t) d t- \\
-\frac{P(x)}{(n-1) !} \sum_{r=1}^{r=n} \frac{1}{\left(x-x_{r}\right) P^{\prime}\left(x_{r}\right)} \int_{\xi}^{x_{r}}\left(x_{r}-t\right)^{n-1} g(t) d t .
\end{gathered}
$$


37. Supposons maintenant que $g(x)$ soit simplement un quotient différentiel $n^{\ominus}$ généralisé $g_{n}(x)$ relatif à un certain système particulier donné de $n+1$ constantes deux à deux distinctes $\alpha_{1}, \alpha_{2}, \ldots, \alpha_{n+1}$. $g_{n}(x)$ vérifie par hypothèse l'égalité (32) par rapport à la fonction inconnue $f(x)$. La fonction de $(n+1)$ variables $V\left(f, x_{1}, \ldots, x_{n+1}\right)=V$ est-elle donnée par l'égalité (33) où $g$ est remplacée par $g_{n}(x)$, tout au moins moyennant certaines hypothèses vérifiées par $g_{n}(x)$, et à la condition d'effectuer les opérations intégrales de (19) suivant une règle convenablement posée?

Les égalités (33), (34), (35) sont effectivement exactes dans le cas très particulier où l'on suppose encore que, sur le segment $a b$ oì les $x_{r}$ sont choisis arbitrairement, le nombre $\left|R_{n}\left(f, x, h, \alpha_{l}\right)\right|$ est borné indépendamment de $x$ et de $h$ (les $\alpha_{t}$ étant indépendants de $x$ et de $h$ et donnés).

La démonstration est celle dont s'est servi H. Lebesgue dans des questions analogues à celle-ci. Posons

$$
\Phi\left(g_{n}, x_{r}\right)=\frac{1}{(n+1) !} \sum_{r=1}^{r=n+1} \frac{1}{\psi^{\prime}\left(x_{r}\right)} \int_{\xi}^{x_{r}}\left(x_{r}-x\right)^{n-1} g_{n}(x) d x .
$$

D'après un théorème fondamental, les conditions:

$$
g_{n}(x)=\lim _{h=0} R_{n}\left(f, x, h, \alpha_{i}\right)
$$

$R_{n}$ borné indépendamment de $x$ et de $h$, entraînent

$$
\begin{gathered}
\Phi\left(g_{n}, x_{r}\right)=\lim _{h=0} \Phi\left(R_{n}, x_{r}\right) . \\
\text { Or, } \\
\Phi\left(R_{n}, x_{r}\right)=\frac{1}{(n-1) !} \sum_{r=1}^{r=n+1} \frac{1}{\psi^{\prime}\left(x_{r}\right)} \int_{\xi}^{x_{r}}\left(x_{r}-x\right)^{n-1} \cdot \frac{n !}{h^{n}}\left[\sum_{i=1}^{i=n+1} \frac{f\left(x+\alpha_{r} h\right)}{\varphi^{\prime}\left(\alpha_{i}\right)}\right] d x= \\
=\frac{n !}{h^{n}} \sum_{i=1}^{i=n+1} \frac{1}{\varphi^{\prime}\left(\alpha_{i}\right)} \cdot \frac{1}{(n-1) !} \sum_{r=1}^{x_{n}+1} \frac{1}{\psi^{\prime}\left(x_{r}\right)} \int_{\xi}^{x_{r}}\left(x_{r}-x\right)^{n-1} f\left(x+\alpha_{i} h\right) d x .
\end{gathered}
$$

Soit $F(x)$ une fonction continue dont la $n^{\theta}$ dérivée est la fonction continue $f(x) . F\left(x+\alpha_{l} h\right)$ a pour dérivée $n^{\ominus} f\left(x+\alpha_{l} h\right)$. D'après la formule (33)

$$
\begin{gathered}
V\left(F, x_{r}+\alpha_{l} h\right)=\sum_{r=1}^{r=n+1} \frac{F\left(x_{r}+\alpha_{i} h\right)}{\psi^{\prime}\left(x_{r}\right)}= \\
=\frac{1}{(n-1) !} \sum_{r=1}^{r=n+1} \frac{1}{\psi^{\prime}\left(x_{r}\right)_{\xi}} \int_{\xi}^{x_{r}}\left(x_{r}-x\right)^{n-1} f\left(x+\alpha_{l} h\right) d x,
\end{gathered}
$$

done

$$
\begin{gathered}
\Phi\left(R_{n}, x_{r}\right)=\frac{n !}{h^{n}} \sum_{i=1}^{i=n+1} \frac{1}{\varphi^{\prime}\left(\alpha_{i}\right)} \sum_{r=1}^{r=n+1} \frac{F\left(x_{r}+a_{i} h\right)}{\psi^{\prime}\left(x_{r}\right)}= \\
=\sum_{r=1}^{r=n+1} \frac{1}{\psi^{\prime}\left(x_{r}\right)} \cdot \frac{n !}{h^{n}} \sum_{i=1}^{i=n+1} \frac{F\left(x_{r}+\alpha_{i} h\right)}{\varphi^{\prime}\left(\alpha_{i}\right)} .
\end{gathered}
$$

$f(x)$ étant la dérivée $n^{0}$ de $F(x)$ en est a fortiori le $n^{\theta}$ quotient généralisé relativement aux constantes $\alpha_{1}, \alpha_{2}, \ldots$ Donc

et enfin

$$
\lim _{h=0} \frac{n !}{h^{n}} \sum_{i=1}^{i=n+1} \frac{F\left(x_{r}+\alpha_{i} h\right)}{\varphi^{\prime}\left(\alpha_{i}\right)}=f\left(x_{r}\right)
$$

$$
\Phi\left(g_{n}, x_{r}\right)=\lim _{h=0} \Phi\left(R_{n}, x_{r}\right)=\sum_{r=1}^{r=n+1} \frac{f\left(x_{r}\right)}{\psi^{\prime}\left(x_{r}\right)}=V\left(f, x_{r}\right) .
$$

Donc, sur tout intervalle où $R_{n}$ est borné indépendamment de $x$ et de $h$, les égalités équivalentes entre elles (33), (34), (35) sont valables.

On pourrait observer que les raisonnements et les conclusions subsistent si l'on considère que l'égalité

$$
\lim _{h=0} R_{n}\left(f, x, h, \alpha_{i}\right)=g_{n}(x)
$$

a lieu non pas quand $h$ tend indifféremment vers 0 , mais simplement quand $h$ tend vers 0 en prenant sur l'intervalle considéré une succession de valeurs $h^{\prime}, \ldots, h^{(m)}$ de signes quelconques, indépendantes de $x$.

38. $f(x)$ étant supposée continue en chaque point, la fouction $g_{n}(x)$ limite de $R_{n}\left(f, x, h, \alpha_{i}\right)$ est continue en $x$. D'après le raisonnement de Baire, l'ensemble des points de $a b$ au voisinage desquels $R_{n}$ n'est pas borné indépendamment de $x$ et de $h$ est non-dense. Soit $K$ cet ensemble, dont on ne peut a priori déduire la position de la seule connaissance de $g_{n}(x)$.

Dans tout intervalle $j$ contigu à l'ensemble inconnu $K$, quels que soient les $x, x_{r}$ situées sur $j$, l'identité (34) est valable, l'intégration se faisant au sens de Lebesgue.

Si donc $g_{n}(x)$ est sommable sur la totalité de $a b$, le second membre de (35) est une fonction $\theta(x)$ telle que la différence $\theta(x)-f(x)$ est un polynome de degré $(n-1)$ au plus dans tous les intervalles contigus da un ensemble fermé non-dense, d'ailleurs inconnu. 
Pour pouvoir espérer $1^{0}$ la validité de (35) dans tout intervalle où $g_{n}(x)$ est borné, $2^{0}$ la possibilité d'étendre la définition de l'intégrale pour que l'égalité (35) soit vraie dans tout intervalle où $g_{n}(x)$ vérifie la condition (32), il faut avant tout que la connaissance de $g_{n}$ détermine les combinaisons équivalentes $V\left(f, x_{r}\right), U\left(f, x_{r}\right)$.

Il faut et il suffit pour cela que, si $g_{n}(x)$ est nul, $f(x)$ soit nécessairement sur la totalite de ab un polynome, de degrd $(n-1)$ au plus.

$38 \mathrm{~b}$. D'après ce que nous avons vu, si $g_{n}(x)=0$ sur $a b$, l'ensemble des points de $a b$ autour desquels $f$ n'est pas un polynome unique d'ordre $n-1$ au plus est un ensemble fermé non-dense' $E$. Il est naturel de rechercher les conditions à vérifier par les $\alpha_{t}$ pour que E ne puisse pas exister.

Nous savons déjà que si la suite $\alpha_{1}, \ldots, \alpha_{n+1}$ ne présente pas les deux signes, $E$ peut exister et renfermer un ensemable fermé quelconque donné d'avance. Nous devons donc supposer les $\alpha_{t}$ non tous de même signe, par exemple:

$$
\alpha_{1}>\alpha_{2}>\ldots>\alpha_{k}>0 \geqslant \alpha_{k+1}>\ldots>\alpha_{n+1} .
$$

Nous appellerons rang de la combinaison $Q_{n}\left(f, x, h, \alpha_{1}, \ldots, \alpha_{n+1}\right)$ le nombre des coefficients $\alpha_{i}$ de même signe qui ne sont pas en majorité.

Le changement de $h$ en $-h$ permet de supposer $n \geqslant 2 k-1$, si $\alpha_{k+1}<0$ et $n \geqslant 2 k$, si $\alpha_{k+1}=0$. k sera dès lors le rang de la combinaison $Q_{n}$.

Le changement de $h$ en $\lambda h$ permet de choisir la valeur d'un coefficient $\alpha_{i}$.

39. Pour que $E$ ne puisse pas exister, il faut d'abord que $E$ ne puisse pas avoir de point isolé.

En un point isolé $c$ de $E, f(x)=P_{n-1}(x)$ pour $x \geqslant c, f(x)=Q_{n-1}(x)$ pour $x \leqslant c, P_{n-1}$ et $Q_{n-1}$ étant deux polynomes différents, de degré $n-1$ au plus. Comme $f$ peut être accru dans toút son champ d'existence d'un polynome quelconque d'ordre $n-1$ au plus, tout revient à exclure la possibilité que, avec $\lim _{h=0} R_{n}=0$, on ait à la fois $f(x)=0$ pour $x \leqslant 0$

et $f(x)=A_{1} x^{n-1}+A_{2} x^{n-2}+\ldots A_{n-1} x=A_{n-1}(x)$ pour $x>0$, les $A_{m}$ n'étant pas tous nuls.
On en conclura la possibilité d'avoir

$$
f(x)=P_{n-1}(x) \text { pour } c^{\prime} \leqslant x \leqslant c
$$

et $f(x)=P_{n-1}(x)+A_{1}(x-c)^{n-1}+\ldots+A_{n-1}(x-c)$ pour $c \leqslant x \leqslant c^{\prime \prime}$, avec les mêmes coefficients non nuls $A_{m}$ que pour $c=0$.

La condition pour que $A_{m} \neq 0$ est

équivalente à

$$
\frac{\alpha_{1}^{m}}{\varphi^{\prime}\left(\alpha_{1}\right)}+\frac{a_{2}^{m}}{\varphi^{\prime}\left(\alpha_{2}\right)}+\ldots+\frac{\alpha_{k}^{m}}{\varphi^{\prime}\left(\alpha_{k}\right)}=0
$$

$$
\sum_{i=k+1}^{i=n+1} \frac{\alpha_{i}^{m}}{\varphi^{\prime}\left(\alpha_{i}\right)}=0
$$

Si (36) est vérifiée, la fonction $f$ égale à 0 d'un côté du point $c$ et à $C(x-c)^{m}$ du côté opposé de $c$, vérifie la condition $\lim _{h=0} R_{n}\left(f, x, h, \alpha_{i}\right)=0$, quels que soient $c$ et $C$ indépendants de $x$.

$39 \mathrm{~b}$. Nous dirons que l'ordre d'indétermination (sous-entendu: de $f$ ) corrélatif à la combinaisoǹ $Q_{n}$ est $q$, s'il existe $q$ exposants $m$ entiers positifs inférieurs à $n$, tels que la fonction $\omega \cdot x^{m}$ où $\omega=0$ pour $x<0, \omega=1$ pour $x>0$, vérifie l'égalité $Q_{n}\left(\omega x^{n}, 0, h, \alpha_{i}\right)=0$, quel que soit $h$.

Pour $x \neq 0$, on a $Q_{n}\left(\omega x^{m}, x, h \alpha_{i}\right)=0$ si $|h|<\mu|x|$, moyennant $\mu \alpha_{1} \leqslant 1,-\mu \alpha_{n+1} \leqslant 1$, puisque dans l'intervalle $0,2 x, \omega x^{n t}$ est un polynome de degré $n-1$ au plus. Par suite $\lim _{h=0} R_{n}\left(\omega x^{m}, x, h, \alpha_{f}\right)=0$, quel que soit $x$.

Les $q$ exposants $m$ vérifiant la condition (36) seront appelés les exposants d'indétermination.

Une combinaison $Q$ dont l'ordre d'indétermination est 0 sera dite caractéristique.

Si $Q_{n}\left(f, x, h, \alpha_{i}\right)$ est caractéristique, $\frac{1}{h^{n-1}} Q_{n}\left(\omega x^{m}, 0, h, \alpha_{i}\right)$ ne peut tendre vers 0 avec $h$ pour aucune des valeurs $1,2, \ldots, n-1$ de $m$.

40. Si $\alpha_{k+1}=0, n \geqslant 2 k$, et si $\mu(x)=x\left(x-\alpha_{k+1}\right) \ldots\left(x-\alpha_{n+1}\right)=$ $=x \theta(x)$, les équations (36) deviennent:

$$
\sum_{i=1}^{i=k} \frac{\alpha_{i}^{m-1}}{\lambda^{\prime}\left(\alpha_{i}\right) \theta\left(\alpha_{i}\right)}=0 \quad(\lambda \mu=\varphi) .
$$

Donc, si la suite $\left(\alpha_{i}\right)$ ne contient pas le coefficient 0 et si $\left(\beta_{j}\right)$ dé- 
signe la suite obtenue en ajoutant le coefficient 0 à la suite des coefficients $\alpha_{t}$, la combinaison $Q_{n}$ relative d̀ la suite $\left(\alpha_{t}\right)$ et la combinaison $Q_{n+1}$ relative à la suite $\left(\beta_{j}\right)$ ont le même ordre d'indétermination. Les exposants d'indétermination de la seconde suite exceddent respectivement d'une unité ceux de la première.

41. Soient $m_{1}, m_{2}, \ldots, m_{q}$ les exposants caractéristiques corrélatiff à la suite $\alpha_{i}$, et $c_{p}$ les points d'un ensemble clairsemé.

On peut entourer chacun des $c_{p}$ d'un intervalle propre, de façon que tout point de l'axe des $x$ soit intérieur à un nombre fini de ces intervalles. On en conclut que les coefficients $C_{p}$.r peuvent toujours être choisis de façon que la série

$$
\Sigma \omega_{p_{1}} C_{p \cdot 1}\left(x-c_{p}\right)^{m_{1}}+\ldots+\Sigma \omega_{p^{\prime} q} C_{p^{\prime} q}\left(x-c_{p}\right)^{m_{q}}
$$

où $C_{p, r} \neq 0, \omega_{p \cdot r}=0$ pour $x<c_{p}, \omega_{p \cdot r}=1$ pour $x>c_{p}$, ait pour somme une fonction f vérifiant la condition $\lim R_{n}\left(f, x, h, \alpha_{l}\right)=0$, quel que soit $x$ sur ab.

42. A priori l'égalité (36) est impossible pour $k=1$. Donc, toute combinaison de rang 1 est caractéristique.

43. Supposons au contraire $k \geqslant 2$ (donc $n \geqslant 3$ si $a_{k+1}>0$ et $n \geqslant 4$ si $\left.\alpha_{k+1}=0\right)$. Remplaçons $\alpha_{k+1}, \alpha_{k+2}, \ldots, \alpha_{k+1}$ par $-\alpha_{k+1}^{\prime},-\alpha_{k+2}^{\prime}, \ldots,-a_{n+1}$, les $\alpha_{s}^{\prime}$ étant positifs et croissants. Posons

$$
\begin{gathered}
\lambda(\alpha)=\left(\alpha-\alpha_{1}\right)\left(\alpha-\alpha_{2}\right) \ldots\left(\alpha-\alpha_{k}\right), \\
\mu(\alpha)=\left(\alpha+\alpha_{k+1}^{\prime}\right)\left(\alpha+\alpha_{k+2}^{\prime}\right) \ldots\left(\alpha+\alpha_{n+1}^{\prime}\right) .
\end{gathered}
$$

La condition (36) devient

(37) $\frac{\alpha_{1}^{m}}{\lambda^{\prime}\left(\alpha_{1}\right) \mu\left(\alpha_{1}\right)}+\frac{\alpha_{2}^{m}}{\lambda^{\prime}\left(\alpha_{2}\right) \mu\left(\alpha_{2}\right)}+\ldots+\frac{\alpha_{k}^{m}}{\lambda^{\prime}\left(\alpha_{k}\right) \mu\left(\alpha_{k}\right)}=0$.

Les nombres $\mu\left(\alpha_{1}\right), \ldots, \mu\left(\alpha_{k}\right)$ sont tous positifs. Les $\lambda^{\prime}\left(\alpha_{1}\right), \ldots, \lambda^{\prime}\left(\alpha_{k}\right)$ sont alternativement positifs et négatifs.

Il est aisé de voir qu'une équation en $r$ :

$$
B_{1} a_{1}^{r}-B_{2} a_{2}^{r}+\ldots+(-1)^{k} B_{k} a_{k}^{r}=0,
$$

où les $B$ sont positifs et les $a_{s}$ décroissent, a au plus $k-1$ racines réelles en $r$. En divisant par $a_{k}^{r}$ et dérivant par rapport à $r$, on trouve en effet une équation du même type, mais possédant un terme de moins, ce qui rend la proposition évidente.

Done, l'ordre d'indétermination d'une combinaison $Q_{n}\left(f, x, h, \alpha_{t}\right)$ est inférieur au rang $k$ de cette combinaison.
44. Quand tous les coefficients $\alpha_{t}$ sont de même signe (l'un d'eux pouvant être nul), nous avons vu que l'ordre d'indétermination de $Q_{n}$ est $n-1$. Voici un autre cas d'indétermination maximum de $f$ connaissant $g_{n}$, le nombre maximum $k-1$ des degrés des termes arbitraires unilatéraux $A_{m}(x-c)^{m}$ étant atteint. C'est le cas oì $k$ étant au moins égal d̀ 2 , le système des nombres $\alpha_{i}$ est symétrique par rapport d̀ l'origine. Donc $\alpha_{n+1-i}=-\alpha_{i}$. On a soit $n=2 k-1$, si $n$ est impair et $\alpha_{k+1}=-\alpha_{k}<0$, soit $n=2 k$ et $\alpha_{k+1}=0$.

Les quotients différentiels généralisés $g_{2 k-1}\left(x, \alpha_{1}, \alpha_{2}, \ldots, \alpha_{k},-\alpha_{k}\right.$, $\left.-\alpha_{k-1}, \ldots,-\alpha_{1}\right)$ et $g_{2 k}\left(x, \alpha_{1}, \ldots, \alpha_{k}, 0,-\alpha_{k}, \ldots,-\alpha_{1}\right)$ ne caracterisent pas $f$ et laissent $\grave{a} f$ une indétermination d'ordre maximum $k-1$.

$$
\begin{aligned}
& \text { Posons } \Psi(u)=\left(u-\alpha_{1}^{2}\right)\left(u-\alpha_{2}^{2}\right) \ldots\left(u-\alpha_{k}^{2}\right) . \\
& \text { Si } n=2 k-1, \quad \varphi(\alpha)=\Psi\left(\alpha^{2}\right), \quad \varphi^{\prime}\left(\alpha_{i}\right)=2 \alpha_{i} \Psi^{\prime}\left(\alpha_{i}^{2}\right) . \\
& \text { Si } n=2 k, \quad \varphi(\alpha)=\alpha \Psi\left(\alpha^{2}\right), \quad \varphi^{\prime}\left(\alpha_{i}\right)=2 \alpha_{i}^{2} \Psi^{\prime}\left(\alpha_{i}^{2}\right) .
\end{aligned}
$$

L'équation (22)

$$
\sum_{i=1}^{l-k} \frac{\alpha_{i}^{m}}{\varphi^{\prime}\left(\alpha_{i}\right)}=0
$$

devient

$$
\sum_{i=1}^{i=k} \frac{\alpha_{i}^{m-1}}{\Psi^{\prime}\left(\alpha_{i}^{2}\right)}=0 \quad \text { si } n=2 k-1, \quad \sum_{i=1}^{i=2} \frac{\alpha_{i}^{m-2}}{\Phi^{\prime}\left(\alpha_{i}^{2}\right)}=0 \text { si } n=2 k \text {. }
$$

Done, si $n=2 k-1$, la condition est satisfaite pour toute valeur impaire de $m$ telle que $\frac{m-1}{2} \leqslant k-2$, où $m=1,3, \ldots, 2 k-3$, ce qui fait bien $k-1$ valeurs de $m$.

Si $n=2 k$, la deuxième condition est satisfaite pour $m$ pair et $\frac{m-2}{2} \leqslant k-2$, donc pour $m=2,4, \ldots, 2 k-2$, ce qui fait encore $k-1$ degrés de termes unilatéraux arbitraires.

Ces résultat s'applique en particulier quand les $\alpha_{t}$ forment une progression arithmétique symétrique par rapport à 0 , auquel cas la combinaison $Q_{n}$ est une différence $n^{\theta}$ de $f(x)$ d'un type classique.

Si $n=2 k-1\left(\alpha_{k+1}<0\right)$, si $n=2 k\left(\alpha_{k+1}=0\right)$, on peut, sans modifier ni l'existence, ni la valeur de $g_{n}\left(f, x, \alpha_{i}\right)$ en aucun point de $a b$, ajouter à $f(x)$ d'un côté et d'un seul du point $c$, dans le premier cas un polynome $I(x-c)$ impair en $x-c$, dans le second cas un polynome $P(x-c)$ pair en $x-c$ et sans terme constant. 
Si l'ensemble elairsemé quelconque $c_{k}$ est donné, on pourra ajouter à $f$ une série de polynomes unilatéraux $\Sigma \omega_{p} C_{p}, I_{p}\left(x-c_{p}\right)$, $\Sigma \omega_{p} C_{p} P_{p}\left(x-c_{p}\right)$ sans changer $g_{n}(f, x)$, les coefficients $C_{p}$ supposés non nuls devant être convenablement choisis, étant donnés les $I$ et les $P_{p}$.

45. Nous ne nous proposerons pas de rechercher toutes les combinaisons non caractéristiques. Bornons-nous à de sommaires indications dans les cas de $k=2$ et $k=3$.

Si $k=2$ (d'où $n \geqslant 3$ si $\alpha_{3}<0, n \geqslant 4$ si $\alpha_{8}=0$ ), l'équation (36) devient

$$
\frac{\alpha_{1}^{m}}{\left(\alpha_{1}+\alpha_{3}^{\prime}\right) \ldots\left(\alpha_{1}+\alpha_{n+1}^{\prime}\right)}=\frac{\alpha_{2}^{n}}{\left(\alpha_{2}+\alpha_{3}^{\prime}\right) \ldots\left(\alpha_{2}+\alpha_{n+1}^{\prime}\right)}
$$

Si $m \geqslant n-1$, la fonction $\frac{x^{m}}{\left(x+\alpha_{8}^{\prime}\right) \ldots\left(x+\alpha_{n+1}^{\prime}\right)}$ est constamment décroissante. L'égalité est impossible si $\alpha_{1} \neq \alpha_{2}$. Donc $m \leqslant n-2$. Si $\alpha_{3}<0, \quad(n \geqslant 3), \quad 1 \leqslant m \leqslant n-2 . \quad$ Si $\alpha_{\mathrm{a}}=0, \quad(n \geqslant 4)$, l'équation est

$$
\frac{\alpha_{1}^{m-1}}{\left(\alpha_{1}+\alpha_{4}^{\prime}\right) \ldots\left(\alpha_{1}+\alpha_{n+1}^{\prime}\right)}=\frac{\alpha^{m-1}}{\left(\alpha_{2}+\alpha_{k}^{\prime}\right) \ldots\left(\alpha_{1}+\alpha_{n+1}^{\prime}\right)}
$$

Done $2 \leqslant m \leqslant n-2$.

En particulier, pour $n=3, \alpha_{3}<0$, il n'y a d'autre solution possible que $m=1$ avec $\alpha_{1} \alpha_{2}=\alpha_{3}^{\prime} \alpha_{4}^{\prime}$, et pour $n=4, \alpha_{3}=0$, la seule solution possible est $m=2$ avec $\alpha_{1} \alpha_{2}=\alpha_{4}^{\prime} \alpha_{b}^{\prime}$.

Done les quotients différentiels suivanț̣, où $0<\beta<\gamma \leqslant 1$,

$$
\begin{gathered}
g_{8}(f, x, 1, \beta \gamma,-\beta,-\gamma)= \\
=\lim _{h=0} \frac{6}{\beta \gamma(1+\beta)(1+\gamma) h^{8}}\left[\frac{\gamma f(x-\beta h)-\beta f(x-\gamma h)}{\gamma-\beta}-\frac{f(x+\beta \gamma h)-\beta \gamma f(x+h)}{1-\beta \gamma}\right] \\
g_{4}(f, x, 1, \beta \gamma, 0,-\beta,-\gamma)= \\
=\lim _{h=0} \frac{24}{\beta^{2} \gamma^{2}(1+\beta)(1+\gamma) h}\left[\frac{\beta^{2} \gamma^{2} f(x+h)-f(x+\beta \gamma h)}{1-\beta \gamma}+\right. \\
\left.+(1+\beta)(1+\gamma) f(x)-\frac{\gamma^{2} f(x+\beta h)-\beta^{2} f(x-\gamma h)}{\gamma-\beta}\right]
\end{gathered}
$$

ne caractérisent pas $f$. Ces quotients différentiels généralisés $g_{3}, g_{k}$ ne sont modifiés ni dans leur existence, ni dans leur valeur par l'addition à $f$ d'une série de foncțions $\Sigma C_{p} \omega_{p}\left(x-c_{p}\right)$ dans le premier cas, $\Sigma C_{p} \omega_{p}\left(x-c_{p}\right)^{2}$ dans le second eas, avec les notations utilisées plus haut.

46. Pour $k=3$ (donc $n \geqslant 5$ si $\alpha_{4}<0, n \geqslant 6$ si $\alpha_{4}=0$ ), on peut chercher des associations de coefficients $\alpha_{t}$ tels que $m$ ait deux valeurs. Il est impossible que ces deux valeurs soient consécutives.

En effet, les deux équations à vérifier seraient, d'après (23),

et

$$
\frac{\alpha_{1}^{m}\left(\alpha_{2}-\alpha_{3}\right)}{\mu\left(\alpha_{1}\right)}+\frac{\alpha_{2}^{m}\left(\alpha_{3}-\alpha_{1}\right)}{\mu\left(\alpha_{2}\right)}+\frac{\alpha_{3}^{m}\left(\alpha_{1}-\alpha_{2}\right)}{\mu\left(\alpha_{3}\right)}=0
$$

Done

$$
\frac{\alpha_{1}^{m+1}\left(\alpha_{2}-\alpha_{3}\right)}{\mu\left(\alpha_{1}\right)}+\ldots=0
$$

$$
\frac{\alpha_{1}^{m}}{\mu\left(\alpha_{1}\right)}=\frac{\alpha_{2}^{m}}{\mu\left(\alpha_{2}\right)}=\frac{\alpha_{3}^{m}}{\mu\left(\alpha_{3}\right)}
$$

ce qui est impossible, si les trois nombres $\alpha_{1}, \alpha_{2}, \alpha_{3}$ sont inégaux, puisque $\frac{x^{m}}{\left(x+\alpha_{4}^{\prime}\right)\left(x+\alpha_{5}^{\prime}\right) \ldots\left(x+\alpha_{n+1}^{\prime}\right)}$ a au plus un changement de sens de variation quand $x$ varie de 0 à $+\infty$.

47. Nous arrêtons ici ces considérations. Nous retiendrons de ce qui précède que, si les $\alpha_{i}$ présentent les deux signes, en général l'ensemble $E$ des points au voisinage desquels deux fonctions $f(x), h(x)$ admettant le même $n^{\theta}$ quotient différentiel généralisé $g_{n}\left(x, \alpha_{i}\right)$ n'ont pas pour différence un polynome unique d'ordre $n-1$ au plus, cet ensemble $E$ ne contient pas de points isolés.

48. Il resterait à prouver que si la combinaison $Q_{n}$ est caractéristique, l'ensemble $E$ n'existe pas. Il est déjà établi que s'il existait, il serait parfait, puisqu'il est fermé et dépourvu de points isolés.

Supposons que $E$ parfait existe. A priori, d'après le raisonnement de Baire, $E$ renferme une portion $e$ sur laquelle $\frac{Q_{n}}{h^{n}}$ est borné indépendamment de $x$ sur $e$ et de $h$. Il suffirait d'établir que, $x$ variant indifféremment entre les extrémités de e, $\frac{Q_{n}}{h^{n}}=R_{n}\left(f, x, h, \alpha_{i}\right)$ est borné (ou tout au moins n'est pas non borné dans tout intervalle contenant des points de $e$ ), donc qu'il est impossible de trouver une double suite $x_{p}, h^{(p)}, h^{(p)}$ tendant vers 0 , de façon que $R_{n}\left(f, x_{p}, h^{(p)}, \alpha_{i}\right)$ croisse indéfiniment quand $p$ croît. 
$x_{p}$ aurait évidemment ses points d'accumulation sur $e$, sans qu'aucun des $x_{p}$ puisse étre sux $e$, à partir d'une valeur assez grande de $p$.

Telle est la première question à élucider si l'on veut résoudre le problème de l'intégration d'un quotient différentiel $n^{\bullet}$ généralisé $g_{n}\left(f, x, \alpha_{t}\right)$ quand la combinaison $Q_{n}$ est caractéristique. Je me borne à signaler ce sujet d'étude.

\section{Totalisation des quotients differentiels d'ordre $n$.}

49. Rappelons d'abord quelques définitions et résultats énoncés ailleurs ${ }^{1}$ ).

Une fonction $f(x)$ est dite résoluble (sous-entendu: du premier ordre) sur le segment $a b$, si elle est continue sur $a b$ et si sa variation sur tout ensemble parfait mince est réductible à zéro.

Ces conditions expriment les propriétés suivantes:

a) [réductibilité de la variation de $f(x)$ sur un ensemble parfait $P$ ]. Quel que soit l'ensemble parfait $P$, dont les contigus sont désignés par $a_{m} b_{m}$ ou $i_{m}$, si $V_{m}=f\left(b_{m}\right)-f\left(a_{m}\right)$ désigne la variation simple de $f(x)$ sur $i_{m}$, l'ensemble des points $\xi$ de $P$ au voisinage desquels la série $\underset{m}{\Sigma}\left|V_{m}\right|$ diverge est non-dense sur $P$.

Ou encore, $P$ contient une portion $P_{1}$ sur laquelle la variation simple de $f(x)$ est définie, ce qui signifie que la série $\Sigma\left|V_{m}\right|$ relative aux contigus de $P_{1}$ converge. La variation simple de $f(x)$ sur $P_{1}$, d'extrémités $\alpha \beta$, est alors par définition $f(\beta)-f(\alpha)-(\alpha \Sigma \beta) V_{m}$.

Il résulte de la çondition a) que l'ensemble $K$ des points de $a b$ au voisinage desquels $f$ n'est pas à variation totale bornée est un ensemble non-dense, évidemment fermé.

b) [réductibilité à 0 de la variation de $f$ sur l'ensemble mince $P$ ]. Si $P$ est de mesure nulle, sur toute portion $P_{1}$ de $P$ où la variation de $f$ est définie, cette variation est nulle.

En particulier, sur tout segment sans point commun avec $K$ $f(x)$ est absolument continue.

Une fonction résoluble possède sur une épaisseur pleine (complémentaire d'un ensemble de mesure nulle ou "mince ${ }^{*}$ ) une dérivée

1) Le lectenr se reportera utilement à mon mémoire sur la dérivation et son calcul inverse, particulièrement aux deux dernières parties parues aux Annales de l'Ecole Normale' Supérieure (1916 et 1917). approximative finie, et connaissant celle-ci $\varphi(x)$, au besoin complétée par une valeur finie quelconque aux points où elle n'existe pas,

$$
f(x)-f(a)=T(\varphi, a, x)
$$

$T(\varphi, a, x)$ étant la totale simple de $\varphi$ entre $a$ et $x$.

50. Nous dirons qu'une fonction $f(x)$ défnie sur un ensemble parfait $Q$ quelconque est résoluble (sous-entendu: du premier ordre) spécialement à cet ensemble $Q$, si $f(x)$ est continue sur $Q$ et si sa variation sur tout ensemble parfait mince $P$ inclus dans $Q$ est réductible à 0 .

Pour que $f$ soit résoluble sur $Q$, il faut et il suffit que la fonetion égale à $f$ sur $Q$ et linéaire sur chaque segment contigu à $Q$ soit résoluble (ou même que toute fonction continue sur $a b$ égale à $f \operatorname{sur} Q$ et résoluble dans chaque contigu à $Q$ soit résoluble sur $a b)$.

$f$ admet sur une pleine épaisseur de $Q$ une dérivée approximative spéciale à $Q$, et a fortiori valable sur le continu, quelle que soit la façon dont $f$ est complétée sur les contigus à $Q$.

Il en résulte que si l'on connaît $1^{\circ}$ pour chaque contigu $\alpha_{m} \beta_{m}$ à $Q$ la différence $f\left(\beta_{m}\right)-f\left(\alpha_{m}\right)$ et en outre $2^{\circ}$ la dérivée approximative $\varphi$ de $f$ sur une pleine épaisseur de $Q$, il suffit de compléter sur les contigus $\alpha_{m} \beta_{m}$ la définition de $\varphi$, en le prenant constant et égal à $\frac{f\left(\beta_{m}\right)-f\left(\alpha_{m}\right)}{\beta_{m}-\alpha_{m}}$ sur $\alpha_{m} \beta_{m}$, pour avoir encore

$$
f(x)-f(\alpha)=T(\varphi, \alpha, x) \text { pour tous les points } x \text { de } Q .
$$

51. Nous dirons qu'une fonction $f(x)$ est résoluble d'ordre $n$ sur un segment $a b$, si elle vérifie les conditions suivantes:

$1^{0}$ En tout point $x$ de $a b$ (conditions locales): 1a) $f(x)$ est continue, $1 \mathrm{~b})$ Quelle que soit la façon dont $h$ non nul tend vers 0 , il est possible de trouver $n$ nombres $h_{1}, h_{2}, \ldots, h_{n}$, de signe opposé à $h$ de valeurs absolues croissantes, pouvant dépendre non seulement de $h$, mais aussi de $x\left[h_{i}=h_{t}(x, h)\right]$, tendant vers 0 avec $h$ (x restant invariable) et tels que, si

$$
\begin{gathered}
\varphi(u)=(u-h)\left(u-h_{1}\right) \ldots\left(u-h_{n}\right), \\
W(f, x, h)=f(x+h)+\varphi^{\prime}(h) \sum_{i=1}^{i=n} \frac{f\left(x+h_{i}\right)}{\varphi^{\prime}\left(h_{i}\right)},
\end{gathered}
$$


la condition

$$
\lim _{h=0} \frac{W(f, x, h)}{h^{n-1}}=0 \text { ou } W(f, x, h)=o\left(h^{n-1}\right) \quad \text { (Laandau) }
$$

53. Supposons connue la variation simple

$f_{n-1}\left(d_{m}, P_{1}\right)-f_{n-1}\left(c_{m}, P_{1}\right)=\theta_{m i}$ de $f_{n-1}\left(x, P_{1}\right)$ sur $c_{m} d_{m}$.

Posons $\mu_{n}(x)=\varphi_{n}(x)$ aux points de $P_{1}$ et $\mu_{n}(x)=\frac{\theta_{m}}{i_{m}}$ pour soit vérifiée.

Cette condition sera satisfaite en particulier quand il existera une combinaison $Q_{n}\left(f, x, h, \alpha_{1}, \alpha_{2}, \ldots, \alpha_{n+1}\right)$ d'ordre $n$, de rang 1 , infiniment petite d'ordre supérieur à $n-1$ par rapport à $h$.

$2^{0}$ (conditions globales relatives à un ensemble parfait $P$, continu ou non). Il existe une portion $P_{1}$ de $P$ en chaque point $x$ de laquelle, spécialement à $P_{1}$, a) $f(x)$ possède une différentielle d'ordre $n-1$,

$f(x+h)=f(x)+h f_{1}\left(x, P_{1}\right)+\ldots+\frac{h^{n-1}}{(n-1) !}\left[f_{n-1}\left(x, P_{1}\right)+\varepsilon_{n-1}(x, h)\right]$

$\varepsilon_{n-1}(x, h)$ tendant vers 0 avec $h, x+h$ ne cessant d'appartenir ì $P$. b) Spécialement à $P_{1}$ et pour $k=1,2, \ldots, n-2, f_{k}\left(x, P_{1}\right)$ admet une différentielle d'ordre $n-k-1$, avec les mêmes quotients différentiels que $f$ :

$f_{k}(x+h)=f_{k}(x)+h f_{k+1}\left(x, P_{1}\right)+\ldots+\frac{h^{n-k-1}}{(n-k-1) !}\left[f_{n-1}\left(x, P_{1}\right)+\varepsilon_{n-1}^{k)}(x, h) \mid\right.$.

c) $f_{n-1}\left(x, P_{1}\right)$ est résoluble sur $P_{1}$.

52. La dérivée approximative de $f_{n-1}\left(x, P_{1}\right)$, spéciale à $P_{1}$, (puisque $f_{n-1}\left(x, P_{1}\right)$ est uniquement défini sur $P_{1}$ ) existe sur une pleine épaisseur de $P_{1}$. C'est un quotient différentiel approximatif $n^{0}$ de $f$.

Donc, les hypotheses $2 \mathrm{a}), 2 \mathrm{c}$ ) entraînent que $f$ admet sur une pleine épaisseur de ab, un quotient différentiel approximatif d'ordre $n$, soit $\varphi_{n}(x)$.

$52 \mathrm{~b}$. Chacun des quotients différentiels spéciaux $f_{k}\left(x, P_{1}\right)(k=1, \ldots, n-2)$ et aussi $f(x)$, ayant spécialement à $P_{1}$ une dérivée continue $f_{k+1}\left(x, P_{1}\right)$, est résoluble surr $P_{1} . f_{k}\left(x, P_{1}\right)$ sera déterminé, à l'addition près d'une constante, par la connaissance de sa variation simple sur chaque segment $c_{m} d_{m}$ ou $i_{m}$ contigu à $P_{1}$ et par la connaissance de $f_{k+1}\left(x, P_{1}\right)$ sur $P_{1}$.

$<x<d_{m}$. Soient $\alpha \beta$ les extrémités de $P_{\mathbf{1}} . \mu_{n}$ est totalisable sur $\alpha \beta$ et $f_{n-1}\left(x, P_{1}\right)-f_{n-1}\left(\alpha, P_{1}\right)=T\left(\mu_{n}, \alpha, x\right)$, quel que soit $x$ sur $P$. Done $f_{n-1}\left(x, P_{1}\right)-f_{n-1}\left(\alpha, P_{1}\right)$ est calculé sur $P_{1}$.

$53 \mathrm{~b}$. Dès lors la connaissance de toutes les variations simples de $f_{n-2}\left(x, P_{1}\right)$ sur chacun des $c_{m} d_{m}$ donnera $f_{n-2}\left(x, P_{1}\right)-f_{n-2}\left(\alpha, P_{1}\right)$. On remontera de proche en proche jusqu'à $f$, dont l'excès sur le polynome

$$
f(\alpha)+(x-\alpha) f_{1}\left(\alpha, P_{1}\right)+\ldots+\frac{(x-\alpha)^{n-1}}{(n-1) !} f_{n-1}\left(\alpha, P_{1}\right)
$$

est déterminé, ce polynome lui-même pouvant être quelconque.

54. Il est évident que toute fonction résoluble d'ordre $n$ l'est a fortiori d'ordre $k$, si $1 \leqslant k \leqslant n$. La somme, le produit de deux fonctions résolubles d'ordre $n$ sont résolubles d'ordre $n$.

55. La définition du caractère de résolubilité d'ordre $n$ spécialement à un ensemble parfait $Q$ quelconque s'obtiendrait par l'extension naturelle du earactère de résolubilité sur le continu. Dans les conditions locales: $1 a$ la continuité de $f(x)$ en tout point de $Q$ s'entend nspécialement à $Q^{4}, 1 b$ les points $x+h, x+h$ doivent être situés sur $Q$. Dans la condition globale relative à un ensemble parfait quelconque $P$, il faut ajouter que $P$ est dans $Q$.

Nous n'aurons pas ici à utiliser cette généralisation.

Observons néanmoins que si $Q$ a un indice fini en tout point, et si $f(x)$ admet spécialement à $Q$, en tout point et an moins d'un côté, deux coefficients différentiels $n^{\text {øs }}$ finis, $f(x)$ est résoluble d'ordre $n$ spécialement à $Q$.

56. Nous allons montrer que toute fonction résoluble d'ordre $n$, est déterminée, à l'addition près d'un polynome arbitraire d'ordre $n-1$, par la connaissance sur une épaisseur pleine de son $n^{9}$ quotisnt différentiel approximatif, soit $f_{n, a}(x)=\varphi_{n}(x)$. 
Tout revient à montrer que si $f_{n, a}(x)=0$ sur une épaisseur pleine, $f(x)$ est un polynome de degré $n-1$ au plus.

L'énoncé est vrai pour $n=1$. Admettons qu'il soit vrai pour tous les ordres inférieurs à $n$ et démontrons-le pour la valeur considérée de $n$.

D'abord, l'ensemble des points au voisinage desquels $f(x)$ n'admet pas un quotient différentiel général d'ordre $n-1$ résoluble est non-dense sur le continu (condition $2 \mathrm{c}$ appliquée à $P$ identique à $a b)$. Ce quotient $f_{n-1}(x)$ ayant la dérivée approximative 0 sur une épaisseur pleine est constant sur tout intervalle où il est résoluble. Si done $f(x)$ n'est pas un polynome unique sur $a . b$, de degré $n-1$ au plus, l'ensemble $E$ des points autour desquels $f$ ne coïncide pas avec un polynome unique est non-dense, $E$ est évidemment fermé.

$E$ n'a pas de point isolé. Car si $E$ possède un tel point $\xi$, de part et d'autre de $\xi, f(x)$ coïncide avec deux polynomes distincts d'ordre $n-1$ au plus, $M(x)$ pour $x>\xi, N(x)$ pour $x<\xi$. Soit $L(x)=M(x)-N(x)$. La fonction $\theta(x)$ égale à 0 pour $x<\xi$, à $L(x)$ pour $x>\xi$, doit vérifier $W(\theta, \xi, h)=o\left(h^{n-1}\right)$. Or $W(\theta, \xi, h)=L(\xi+h)$ si $h>0 . W(\theta, \xi, h)=\varphi(h) \sum \frac{L\left(\xi+h_{i}\right)}{\varphi^{\prime}\left(h_{t}\right)}=L(\xi+h)$ si $h<0$. Donc $L(\xi+h)=o\left(h^{n-1}\right) . \quad L$ étant de degré $n-1$ au plus, $L(x)$ est identiquement nul. $N(x)=M(x)$. $\xi$ n'appartient pas à $E$. $\quad E$ n'a pas de point isolé.

$E$, s'il existe, est donc parfait. Il possède une portion $E_{1}$ sur laquelle les hypothèses $2^{0}$ sont vérifiées. Soit $c d$ ou $i$ un intervalle contiga à $E$. Sur $c d, f$ est un polynome $P(x)$ de degré au plus égal à $n-1$.

Soit $h$ négatif et assez petit pour que $h_{n}=h_{n}(c, h)$, qui est positif, soit inférieur à $i$. Alors $c+h_{r}$ est sur $i$, pour $r=1,2, \ldots, n$ et $f\left(c+h_{r}\right)=P\left(c+h_{r}\right)$. Mais

$$
\frac{P(c+h)}{\varphi^{\prime}(h)}+\sum_{r=1}^{r=n} \frac{P\left(c+h_{r}\right)}{\varphi^{\prime}\left(h_{r}\right)}=0,
$$

$P$ étant un polynome de degré $n-1$ au plus. Donc

$$
W(f, c, h)=f(c+h)-P(c+h)=o\left(h^{n-1}\right) \quad \text { si } \quad h<0 .
$$

Et de même

$$
f(d+h)-P(d+h)=o\left(h^{n-1}\right) \text { si } h>0 .
$$

Mais, $x$ étant sur $E_{1}$,

$$
\begin{gathered}
f(x)=f(c)+(x-c) f_{1}\left(c, E_{1}\right)+\ldots+\frac{(x-c)^{n-1}}{(n-1) !}\left[f_{n-1}\left(x, E_{1}\right)+\varepsilon\right]= \\
=P(x)+o(x-c)^{n-1} .
\end{gathered}
$$

Le même raisonnement s'applique au point $d$.

On en conclut l'identité avec $P(x)$ des deux polynomes:

$$
\begin{aligned}
& f(c)+(x-c) f_{1}\left(c, E_{1}\right)+\ldots+\frac{(x--c)^{n-1}}{(n-2) !} f_{n-1}\left(c, E_{1}\right), \\
& f(d)+(x-d) f_{1}\left(c, E_{1}\right)+\ldots+\frac{(x-d)^{n-}}{(n-1) !} f_{n-1}\left(d, E_{1}\right) .
\end{aligned}
$$

En particulier $f_{n-1}\left(d . E_{1}\right)=f_{n-1}\left(c, E_{1}\right)$. Donc la variation de $f_{n-1}\left(x, E_{1}\right)$ entre les extrémités de chaque contigu à $E_{1}$ est nulle.

Considérons la fonction $\mu_{n}(x)$ ainsi définie sur le segment $\alpha \beta$ joignant les extémités de $E_{1}: \mu_{n}(x)=f_{n-1}\left(x, E_{1}\right)$ si $x$ est sur $E$ $\mu_{n}(x)=f_{n-1}\left(c, E_{1}\right)=f_{n-1}\left(d, E_{1}\right)$ sur lintervalle $c d$ contigu d̀ $E_{1}$.

$\mu_{n}(x)$ est continu, résoluble d'ordre 1 et a sur une pleine épaisseur de $\alpha \beta$ la dérivée approximative 0 . Donc $\mu_{n}(x)$ est constant entre les extrémités de $E_{1}$. Soit $A_{n-1}$ sa valeur. Posons

$$
m_{1}(x)=f(x)-A_{n-1} \frac{x^{n-1}}{(n-1) !} .
$$

$m_{1}(x)$, résoluble d'ordre $n-1$ sur $\alpha \beta$, y possède sur une épaisseur pleine le $(n-1)^{\bullet}$ quotient différentiel approximatif 0 . Donc, d'après ce que nous avons admis, $m_{1}(x)$ est un polynome d'ordre $n-2$ au plus sur $\alpha \beta$. Done $f$ est un polynome unique d'ordre $n-1$ au plus sur $\alpha \beta$. Done $E$ n'a aucun point sur lintervalle $\alpha \beta$, contrairement à l'hypothèse que $E_{1}$ est une portion de $E$. Nous aboutissons à une contradiction. Le théorème est établi.

57. Il résulte de ce théorème que, si une suite d'opérations appliquée à $\varphi_{n}(x)$ nous donne sur $a b$ une fonction $m(x)$ résoluble d'ordre $n$ et admettant sur une épaisseur pleine $\varphi_{n}$ pour dérivée approximative d'ordre $n$, toute fonction $f n$ fois résoluble sur $a b$, admettant $\varphi_{n}(x)$ pour dérivée approximative $n^{\mathrm{e}}$ sur une épaisseur pleine, est la somme de $m(x)$ et d'un polynome d'ordre $n-1$ au plus. Cette observation justifie la légitimité da calcul suivant de $f(x)$ à l'aide de $\varphi_{n}$.

Fundamenta Mathematicae T. XXV. 
58. $f$ inconnu étant résoluble, il y a (2c) par hypothèse, sur tout intervalle de $a b$, un segment où $f_{n \rightarrow-1}$ existe, est continu et est résoluble. Sur ce segment $\varphi_{n}(x)$ est totalisable. On en tire pour $\varphi_{n}$ ce

Caractère (2c). Si E' est l'ensemble des points qui ne sont intérieurs à aucun intervalle sur lequel $\varphi_{n}$ est totalisable, $E^{\prime}$ est non-dense.

Soit $i$ un intervalle contigu à $E$. Sur tout segment intérieur à $i$ $\varphi_{n}$ est totalisable. Soit $\alpha$ un point intérieur à $i$. Posons

$$
I(x)=\frac{1}{(n-1) !} T\left[(x-t)^{n-1} \varphi_{n}(t), \alpha, x\right]
$$

$x$ étant un point quelconque intérieur à $i$. Le second membre est une fonction continue dans $i$, ayant ses $n-1$ dérivées premières continues, la $(n-1)^{\theta}$ étant $T\left[\varphi_{n}(t), \alpha, x\right]$. Oelle-ci est résoluble et a pour dérivée approximative $\varphi_{n}(x)$ sur une épaisseur pleine. Done $f(x)-I(x)$ est un polynome $P(x)$ sur la totalité de $i$.

59. Rappelons incidemment que, en un ensemble partout épais sur $i, \varphi_{n}$ est la dérivée ordinaire de $T\left(\varphi_{n}, \alpha, x\right)$. En un point de cet ensenable, $\varphi_{n}$ est la dérivée ordinaire $n^{\ominus}$ de $f(x)$. Supposons que $\alpha$ soit dans cet ensemble. $I(x)$ est nul ainsi que ses $n-1$ premières dérivées au point $\alpha$ et sa dérivée $n^{\ominus}$ ordinaire y est égale à $\varphi_{n}(\alpha)$.

Au point $\alpha$, les valeurs de $P(x)$ et de ses $n-1$ premières dérivées sont celles de $f(x)$.

60. Soient $\gamma, \delta$ les extrémités de $i . x$ se déplaçant sur l'intervalle $i, f(x)$ tend vers une limite unique (condition $1 a$ ) quand $x$ tend vers $\gamma$ et il en est de même quand $x$ tend vers $\delta$. Il en est done de même pour $I(x)$, et c'est là un caractère nécessaire (caractère 1a) que doit presenter $\varphi_{n}$.

Mais, bien entendu, même si $f$ possède au point $\gamma$ ou au point $\delta$ des quotients différentiels des $n-1$ premiers ordres, il n'en résulte nullement que $I^{(k)}(x)=\frac{1}{(n-k-1) !} T\left[(x-t)^{n-k-1} \varphi(t), \alpha, x\right]$ tende vers une limite quand $x$ tend vers $\gamma$ ou vers $\delta$, même pour $k=1$

61. Convenons de dire que le problème de la détermination de $f(x)$ sur un intervalle $u$ est résolu, si nous connaissons $f(x)$ sur $u$ à l'addition près d'un polynome unique pour tout l'intervalle u. Nous saurons résoudre le problème pour la totalité de $a b$, si nous savons résoudre chacun des deux suivants:
Problème A. Ayant déterminé $f(x)$ séparément sur deux intervalles adjacents $i, j$ séparés par leur extrémité commune $\xi$, déterminer $f$ sur l'intervalle $i+\xi+j$.

Problème B. Ayant déterminé $f(x)$ sur chaque intervalle contigu à un ensemble parfait totalement discontinu $P$, déterminer $f$ tout $a ̈ u$ moins sur un inter valle contenant des points de $P$.

62. Problème A. Sur $i$, nous avons $f(x)=I(x)+M(x) ; \operatorname{sur} j$, $f(x)=J(x)+N(x) ; I(x)$ et $J(x)$ sont deux fonctions continues au point $\xi$, et $M(x), N(x)$ sont deux polynomes de degré $n-1$ au plus, arbitraires jusqu'ici. L'un de ces polynomes, $M(x)$ par exemple, restant arbitraire, il s'agit de relier le second $N(x)$ au premier, c'est-à-dire de montrer que la différence $L(x)=N(x)-M(x)$ est déterminée.

Nous utiliserons la propriété $1 b$ de $f(x)$. Supposons $i$ à gauche de $\xi$. Faisant $h_{r}=h_{r}(\xi, h)$ et supposant $h$ assez petit pour que $h_{n}$ soit dans $i$, si $0<h<j$, et dans $j$, si $-i<h<0$, considérons $W(f, \xi, h)=o\left(h^{n-1}\right)$.

Si $h>0$,

$f(\xi+h)=J(\xi+h)+N(\xi+h)_{,} f\left(\xi+h_{r}\right)=I\left(\xi+h_{r}\right)+M\left(\xi+h_{r}\right)_{r}$

$$
\sum_{r=1}^{r=n} \frac{f\left(\xi+h_{r}\right)}{\varphi^{\prime}\left(h_{r}\right)}=\sum_{r=1}^{r=n} \frac{I\left(\xi+h_{r}\right)}{\varphi^{\prime}\left(h_{r}\right)}-\frac{M(\xi+h)}{\varphi^{\prime}(h)} .
$$

Posons

$$
\varphi^{\prime}(h) \sum_{r=1}^{r=n} \frac{I\left(\xi+h_{r}\right)}{\varphi^{\prime}\left(h_{r}\right)}=W_{1}(I, \xi, h) .
$$

$W_{1}(I, \xi, h)$ est une fonction connue de $h$ positif suffisamment petit. On a

$J(\xi+h)+W_{1}(I, \xi, h)+N(\xi+h)-M(\xi+h)=o\left(h^{n-1}\right) \quad(h>0)$. De même

$I(\xi+h)+W_{1}(J, \xi, h)+M(\xi+h)-N(\xi+p)=o\left(h^{n-1}\right) \quad(h<0)$.

Donc (caractère 16 de $\varphi_{n}$ ): la fonction de $h$ égale à

$-J(\xi+h)-W_{1}(l, \xi, h)$ pour $h<0$ et à $I(\xi+h)+W_{1}(J, \xi, h)$ pour $h>0$, admet une différentielle générale d'ordre $n-1$ pour $h=0$. D'autre part cette fonction vaut $L(\xi+h)+o\left(h^{n-1}\right)$. 
Done $L(x)$, polynome d'ordre $n-1$ au plus, est parfaitement déterminé et nous venons de donner le moyen de l'obtenir.

Done $f(x)$ est connu sur $i+\xi+j$.

L'application de cette méthode permettra, quand $f(x)$ sera connu sur tout intervalle contigu à un ensemble fermé $E$, de réduî̀e celui-ci $\grave{a}$ son noyau parfait. En effet, par une succession transfinie d'opém. rations du type précédent, $f(x)$ sera connu sur tout intervalle où $E$ est réductible.

63. Problème B. Supposons $f(x)$ connu sur tout intervalle contigu à $P$ parfait, D'après la condition $2^{0}$ il existe une portion $P_{1}$ de $P\left(P_{1}\right.$ est provisoirement inconnu) où $f_{n-1}\left(x, P_{1}\right)$ existe, est réso. luble, avec la dérivéo approximative $\varphi_{n}(x)$ sur une pleine épaisseur de $P_{1}, f_{k}\left(x, P_{1}\right)$ étant résoluble du $k^{\circ}$ ordre sur $P_{1}$, et arec les mêmes quotients différentiels spéciaux à $P_{1}$ que $f(x)$.

Soient $c_{1} d_{1}$ ou $i_{1}, \ldots, c_{m} d_{m}$ ou $i_{m}, \ldots$ les contigus à $P_{1}$ et $c d$ le segment des points extrêmes de $P_{1}$.

Sur $c_{m} d_{m}, f(x)=I_{m}(x)+N_{m}(x) . I_{m}(x)$ est une fonction connue déjà calculée à l'aide de $\varphi_{n} . N_{m}(x)$ est un polynome quelconque de degré $n-1$. Il s'agit de calculer $N_{m}(x)-N_{1}(x)$ quel que soit $m$, et on aura $f(x)$ sur tout le segment $c d$.

Soit $h$ assez petit pour que $h_{n}\left(c_{m}, h\right)(h<0)$ et $\left|h_{n}\left(d_{m}, h\right)\right|$ $(h>0)$ soient inférieurs à $i_{m}=d_{m}-c_{m}$. Oomme dans le cas $\varphi_{n}(x)=0$, nous trouvons

$$
(h<0) f\left(c_{m}+h\right)-N_{m}\left(c_{m}+h\right)+W_{1}\left[I_{m}, c_{m}, h\right]=o\left(h^{n-1}\right)
$$

et

$$
W_{1}\left(I_{m}, c_{m}, h\right)=\varphi^{\prime}(h) \sum_{r=1}^{r=n} \frac{\varphi\left(c_{m}+h_{r}\right)}{\varphi^{\prime}\left(h_{r}\right)}
$$

$$
f\left(d_{m}+h\right)-N\left(d_{m}+h\right)+W_{1}\left(I_{m}, d_{m}, h\right)=o\left(h^{n-1}\right) \quad(h>0) .
$$

Supposons $c_{m}+h$ situé sur $P_{1}$. On a dès lors

$f\left(c_{m}+h\right)=f\left(c_{m}\right)+h f_{1}\left(c_{m}, P_{1}\right)+\ldots+\frac{h^{n-1}}{(n-1) !} f_{n-1}\left(c_{m}, P_{1}\right)+o\left(h^{n-1}\right)$.

Donc, $W_{1}\left(I_{m}, c_{m}, h\right)$ admet spécialement à l'ensemble décrit par $h$ quand $c_{m}+h$ décrit $P_{1}$, une différentielle d'ordre $n-1$ pour $h=0$, soit $W_{1}\left(I_{m}, c_{m}, h\right)=\omega_{0}+\omega_{1} h+\ldots+\omega_{n-1} \frac{h^{n-1}}{(n-1) !}+o\left(h^{n-1}\right)$.

\section{Donc}

$$
\begin{gathered}
N_{m}(x)= \\
\quad f\left(c_{m}\right)+\omega_{0}+\left(x-c_{m}\right)\left[f_{3}\left(c_{m}, P\right)+\omega_{1}\right]+\ldots \\
\ldots+\frac{(x-c)^{n-1}}{(n-1) !}\left[f_{n-1}\left(c_{m}, P_{1}\right)+\omega_{n-1}\right]
\end{gathered}
$$

et de même $W_{1}\left(I_{m}, d_{m}, h\right)$ admet spécialement à l'ensemble décrit par $h$ quand $d_{m}+h$ décrit $P_{1}$ une différentielle d'ordre $n-1$ au point $h=0$ et vaut par conséquent

$$
\omega_{0}^{\prime}+\omega_{1}^{\prime} h+\ldots+\omega_{n-1}^{\prime} \frac{h^{n-1}}{(n-1) !}+o\left(h_{n-1}\right) .
$$

Donc encore

$$
\begin{gathered}
N_{m}(x)=f\left(d_{m}\right)+\omega_{0}^{\prime}+\left(x-d_{m}\right)\left[f_{1}\left(d_{m}, P_{1}\right)+\omega_{1}^{\prime}\right]+\ldots \\
\ldots+\frac{\left(x-d_{m}\right)^{n-1}}{(n-1) !}\left[f_{n-1}\left(d_{m}, P_{1}\right)+\omega_{n-1}^{\prime}\right] .
\end{gathered}
$$

En égalant les deux expressions de $N_{m}(x)$, on voit que $f_{k}\left(d_{m}, P_{1}\right)$ s'exprime linéairement au moyen de $f_{k}\left(c_{m}, P_{1}\right)$ :

$$
\begin{gathered}
f_{n-1}\left(d_{m}, P_{1}\right)-f_{n-1}\left(c_{m}, P_{1}\right)=\omega_{n-1}-\omega_{n-1}^{\prime}=\theta_{m, n-1}, \\
f_{k}\left(d_{m}, P_{1}\right)+\omega_{k}^{\prime}=f_{k}\left(c_{m}, P\right)+\omega_{k}+\left(d_{m}-c_{m}\right)\left[f_{k+1}\left(c_{m}, P_{1}\right)+\omega_{1}\right]+\ldots \\
\ldots+\frac{\left(d_{m}-c_{m}\right)^{n-k-1}}{(n-k-1) !}\left[f_{n-1}\left(c_{m}, P_{1}\right)+\omega_{n-1}\right], \\
(k=0,1, \ldots, n-1) .
\end{gathered}
$$

$63 \mathrm{~b}$. Nous trouvons donc que le calcul appliqué à $\varphi_{n}$ doit mettre en évidence les caractères suivants:

Caractère $(2 a)$. L'ensemble $K_{0}$ des points de $P$ au voisinage desquels existent des intervalles contigus $c_{m} d_{m} \grave{a} P_{1}$ aux extrémités desquels les deux fonctions $W_{1}\left(I_{m}, c_{m}, h\right)$ et $W_{1}\left(I_{m}, d_{m}, h\right)$ n'admettent pas de différentielles $n^{\mathrm{es}}$ respectivement spéciales aux ensembles décrits par $h$ quand $c_{m}+h$ et $d_{m}+h$ décrivent $P$, cet ensemble $K_{0}$ est non-dense sur $P$.

Nous connaissons la variation

$$
f_{n-1}\left(d_{m}, P\right)-f_{n-1}\left(c_{m}, P\right)=\omega_{m, n-1}^{\prime}-\omega_{m, n-1}=\theta_{m, n-1}
$$

pour tout intervalle contigu à $P$ n'ajant aucune de ses extrémités sur $K_{0}$. Sur toute portion $\bar{\omega}$ de $P$ sans point commun avec $K_{0}$, nous pouvons, connaissant les $\theta_{m, n-1}$ relatifs aux contigus à cette portion, tenter la totalisation de $\varphi_{n}$ sur $\bar{\omega}$. 
Caractère $(2 b)$. L'ensemble $H_{t-1} \geqslant K_{0}$ des points au voisinage desquels $\varphi_{n}$ est non totalisable sur $P$, quand on attribue pour variation it sa totale sur le contigu $c_{n t} d_{m}$ is $P$ la valeur $\theta_{n, n-1}, H_{n-1}$ est non-dense sur $P$.

Soit $\bar{\omega}$ une portion de $P$ sans point commun avec $H_{n-1}, \alpha \beta$ les extrémités de $\bar{\omega}$. La fonction $\mu(x)$, égale à $\varphi_{n}$ sur $\bar{\omega}$, à $\frac{\theta_{m}}{i_{n t}}$ sur lo contigu $i_{m}$ à $\bar{\omega}$, est totalisable. Sa totale entre $\alpha$ et $x$ ne diffère que par une constante de $f_{n \rightarrow 1}(x, P)$ sur $\bar{\omega}$. Dès lors, on connâ̂t de la fonction $f_{n-2}(x, P) 1^{0}$ la dérivée approximative égale à $f_{n-1}(x, P)$ sur une pleine épaisseur de $P-H_{n-1}, 2^{0}$ les variations de $f_{n-1}(x, P)$ sur chaque contigu à $P$ n'ayant aucune de ses extrémités sur $H_{n-1-1}$.

63 c. $f_{n \rightarrow 2}(x, P)$ étant résoluble d'ordren $n \ldots 1$, l'ensemble $H_{n \rightarrow 2} \geqslant H_{n-1}$ des points où sa dérivée approximative $f_{n-1}\left(x, P^{\prime}\right)$ n'est pas totulisable est non-dense sur $P$. Sur tout intervalle contigu à $H_{t-2}$, une totalisation simple nous donnera $f_{n-2}(x, P)$, a une fonction linéaire additive près, en tenant compte de la constante arbitraire ajoutable à $f_{n-1}(x, P)$.

De proche en proche, on aboutira à un ensemble $H \geqslant H_{1} \geqslant \ldots \geqslant H_{n-1}$, $H$ étant non-dense sur $P$ et $f(x)$ étant connu à l'addition près d'un polynome de degré $n-1$ sur chaque contigu à $H$,

Par le caractère $1 a$ de la continuité de cette dernière totale, en tout point, on a $f(x)$ sur tout segment contigu à $H$.

64. On conclut de là, comme dans le cas de la totalisation simple, que, par une suite dénombrable d'opérations, on résout le problème de l'intégration de $\varphi_{n}$ pour la totalité du segment $a c$.

65. Dans le cas où la fonction donnée inconnue $f(x)$ est supposée posséder en chaque point de $a b$ une différentielle d'ordre $n$, elle est résoluble d'ordre $n$, d'après le théorème II. Done, on pas. sera, par l'opération que nous venons de décrire, de son $n^{\theta}$ quotient différentiel ordinaire $f_{n}(x)$ supposé donné à la fonction $f(x)$ supposée inconnue.

La même conclusion vaut si $f(x)$ possède en tout point, au moins d'un côté, deux coefficients différentiels $n^{\text {os }}$ extrèmes finis, et ai $\omega_{n}(x)$ est en chaque point égal à l'un d'eux.

\section{Sur l'existence des continus indécomposables.}

$\mathrm{Pa}$

\section{Stefan Mazurkiewicz (Warszawa).}

Je me propose de démontrer que tout espace métrique compact de dimension $\geqq 2$ contient un continu indécomposable $\mathrm{1}$ ).

Désignons par $z$ la variable complexe, par $S_{1}$ la circonférence $|z|=1$, par $Q_{2}$ le disque $|z| \leqq 1$, par $Y^{X}$ la famille des fonctions continues qui transforment l'espace $X$ en sous-ensembles de l'espace $Y$.

Lemme $I^{2}$ ). Si $f$ est une transformation essentielle d'un espace métrique et compact $A$ en $Q_{2}$, alors $f$ est une transformation essentielle de $A_{1}=f^{-1}\left(S_{\mathbf{1}}\right)$ en $S_{\mathbf{1}}$.

Désignons par $x$ les points de $A$, par $u$ les points de $A_{1}$, par $\Gamma_{1}$ la famille des $\varphi \in S_{1}^{A_{1}}$ qui sont inessentielles, par $\Gamma_{2}$ la famille des $\psi \in S_{1}^{A_{1}}$, qui sont extensibles sur $A$ relativement à $S_{1}$. Posons $\varphi_{0}(u)=1$; il vient $\varphi_{0} \in \Gamma_{1}$ et $\varphi_{0} \in \Gamma_{2}$. Or, $\Gamma_{1}$ est conneze et $\Gamma_{2}$, d'après un théorème de $\mathrm{M}$. K. B orsuks), ouvert et fermé. Donc $\Gamma_{1} \subset \Gamma_{2}$.

Supposons que la transformation $f$ de $A_{1}$ en $S_{1}$ soit inessentielle, c. à d. que $f \in \Gamma_{1}$; donc $f \in \Gamma_{2}$ et il existe une fonction $g \in S_{1}^{A}$ telle que $g(u)=f(u)$.

Posons: $f_{t}(x)=(1-t) f(x)+t \cdot g(x), 0 \leqq t \leqq 1$.

On aura: $f_{0}(x)=f(x) ; f_{1}(x)=g(x) \in S_{1} ; f_{t}(x) \in Q_{2} ; f_{t}(u)=f(u)$. Donc, la transformation $f$ de $A$ en $Q_{2}$ est inessentielle, contrairement

à l'hypothèse.

En utilisant un lemme de M. Eilenberg ${ }^{4}$ ), on obtient le

1) Solution d'un problème posé par. M. P. Alexandroff.

2) Les lemmes I, II et le théorème I s'6́tendent aux transformations essentielles en éléments $n$-dimensionnels.

s) B orsuk, Monatsh, f. Math. Phys, 38 (1931), p. 382-383

4) Fund. Math. XXIV, p. 16a-165. 